\title{
Predictive Sliding Mode Control for Attitude Tracking of Hypersonic Vehicles Using Fuzzy Disturbance Observer
}

\author{
Xianlei Cheng, Guojian Tang, Peng Wang, and Luhua Liu \\ College of Aerospace Science and Engineering, National University of Defense Technology, Changsha 410073, China \\ Correspondence should be addressed to Xianlei Cheng; xianleicheng@163.com
}

Received 7 July 2014; Accepted 29 October 2014

Academic Editor: Chunyu Yang

Copyright ( 2015 Xianlei Cheng et al. This is an open access article distributed under the Creative Commons Attribution License, which permits unrestricted use, distribution, and reproduction in any medium, provided the original work is properly cited.

\begin{abstract}
We propose a predictive sliding mode control (PSMC) scheme for attitude control of hypersonic vehicle (HV) with system uncertainties and external disturbances based on an improved fuzzy disturbance observer (IFDO). First, for a class of uncertain affine nonlinear systems with system uncertainties and external disturbances, we propose a predictive sliding mode control based on fuzzy disturbance observer (FDO-PSMC), which is used to estimate the composite disturbances containing system uncertainties and external disturbances. Afterward, to enhance the composite disturbances rejection performance, an improved FDO-PSMC (IFDO-PSMC) is proposed by incorporating a hyperbolic tangent function with FDO to compensate for the approximate error of FDO. Finally, considering the actuator dynamics, the proposed IFDO-PSMC is applied to attitude control system design for HV to track the guidance commands with high precision and strong robustness. Simulation results demonstrate the effectiveness and robustness of the proposed attitude control scheme.
\end{abstract}

\section{Introduction}

Near space is the airspace of Earth altitudes from $20 \mathrm{~km}$ to $100 \mathrm{~km}$, which has shown strategy and spatial superiority with the prosperous development of aerospace technology [1]. The near space hypersonic vehicle (HV) which has many outstanding advantages such as large flight envelope, high maneuverability, and fast response ability compared with the ordinary aircraft has attracted a growing worldwide interest $[2,3]$. Due to the hypersonic velocity and changeable flight environment, the $\mathrm{HV}$ possesses some distinct dynamic characters of highly coupled control channels, serious nonlinearity, and strong uncertainty, which all contribute to the design difficulty of the attitude control system with remarkable precision and strong robustness. Due to the poor performance of the traditional control approaches in addressing the nonlinear and uncertain problem, advanced control methods should be employed in the attitude control system design for the HV, which is still an open problem.

The sliding mode control (SMC) is insensitive to system uncertainties and disturbances. It is one of the most important approaches to the control of systems with modeling imprecision, and it has been widely applied to the flight control system design [4-11]. References [12,13] incorporate the sliding mode surface into the quadratic performance index of the predictive control and then the predictive sliding mode control law which has an explicitly analytical form is derived by minimizing the performance index. By using this method, the undesirable chattering phenomenon is attenuated and the large online computational issue of the predictive control is avoided. The predictive sliding mode control (PSMC) takes merits of the strong robustness of sliding model control and the outstanding optimization performance of predictive control, which appears to be a very promising control method in control engineering.

For the design of control system for the HV, many advanced control methods mainly focus on the stability or robust stability rather than considering the system uncertainties and disturbances explicitly in the controller design [14-18]. They may encounter some unexpected problems and the flight control system may even become unstable in the presence of strong disturbances. Thus, it is of profound significance to adopt new strategies to eliminate the influence of the composite disturbances and improve the precision and robustness of the flight control system. Fuzzy disturbance observer (FDO) is a particular type of disturbance observer, 
which combines the distinct merits of fuzzy control with disturbance observer technology. These advantages make it an appropriate candidate for robust control of uncertain nonlinear systems [19-25]. Nevertheless, the robust flight control scheme based on FDO needs to be further researched for the HV to improve the control ability, which needs to duel with the changeable flight environment and problems caused by system uncertainties.

Motivated by the precise and robust attitude control demand of the HV with uncertain model and external disturbances, this paper considers the composite disturbances in flight control system design of the $\mathrm{HV}$ to improve the robust control performance. Three major contributions are presented as follows.

(1) We propose a predictive sliding mode control based on fuzzy disturbance observer (FDO-PSMC) method for a class of uncertain affine nonlinear systems with system uncertainties and external disturbances. The composite disturbances are considered, which result in poor performance and instability of the control system.

(2) To address the problems which are brought by the composite disturbances, an improved FDO (IFDO) is proposed through utilizing the special properties of a hyperbolic tangent function to compensate for the approximate error of FDO. By using IFDO, the composite disturbances can be approximated effectively.

(3) Considering the actuator dynamics, we apply the improved fuzzy disturbance observer based predictive sliding mode control (IFDO-PSMC) scheme to the attitude control system design for the HV. Numerical simulation verifies the surpassing performance of the proposed control scheme.

The rest of this paper is organized as follows. In Section 2, the control-oriented hypersonic flight model during cruise phase is presented. In order to approximate the composite disturbances, Section 3 presents a FDO-PSMC method and the FDO is improved for a better performance for a class of uncertain affine nonlinear systems with external disturbances. In Section 4, the proposed control scheme is applied to the attitude control system design for the $\mathrm{HV}$ in consideration of the actuator dynamics. Simulation results are presented in Section 5 to validate the effectiveness of the designed flight control system. Finally, Section 6 provides some conclusions of this paper.

\section{HV Modeling}

Suppose that the fuel slosh is not considered and the products of inertia are negligible [26]. Based on the hypothesis of an inverse-square-law gravitational model, the centripetal acceleration for the nonrotating Earth, the mathematical model of $\mathrm{HV}$ during the cruise phase can be described as

$$
\begin{gathered}
\dot{v}=-\frac{\mu}{r^{3}} C_{1}-\frac{D}{m}+\frac{T}{m} \cos \alpha \cos \beta, \\
\dot{\theta}=\frac{1}{m v}\left(-m \frac{\mu}{r^{3}} C_{2}+L \cos \gamma_{V}-N \sin \gamma_{V}+T C_{4}\right), \\
\dot{\sigma}=\frac{-1}{m v \cos \theta}\left(-m \frac{\mu}{r^{3}} C_{3}+L \sin \gamma_{V}+N \cos \gamma_{V}+T C_{5}\right), \\
\dot{\varphi}=\omega_{y} \sin \gamma+\omega_{z} \cos \gamma, \\
\dot{\psi}=\left(\omega_{y} \cos \gamma-\omega_{z} \sin \gamma\right) \sec \varphi, \\
\dot{\gamma}=\omega_{x}-\left(\omega_{y} \cos \gamma-\omega_{z} \sin \gamma\right) \tan \varphi, \\
\dot{\omega}_{x}=J_{x}^{-1} M_{x}+J_{x}^{-1}\left(J_{y}-J_{z}\right) \omega_{z} \omega_{y}, \\
\dot{\omega}_{y}=J_{y}^{-1} M_{y}+J_{y}^{-1}\left(J_{z}-J_{x}\right) \omega_{x} \omega_{z}, \\
\dot{\omega}_{z}=J_{z}^{-1} M_{z}+J_{z}^{-1}\left(J_{x}-J_{y}\right) \omega_{y} \omega_{x},
\end{gathered}
$$

$\sin \beta$

$$
\begin{gathered}
=(\sin (\psi-\sigma) \cos \gamma+\sin \varphi \sin \gamma \cos (\psi-\sigma)) \cos \theta \\
-\cos \varphi \sin \gamma \sin \theta, \\
\sin \alpha \cos \beta=\cos (\psi-\sigma) \sin \varphi \cos \gamma \cos \theta \\
-\sin (\psi-\sigma) \sin \gamma \cos \theta \\
-\cos \varphi \cos \gamma \sin \theta,
\end{gathered}
$$

$$
\begin{gathered}
\sin \gamma_{V} \cos \beta \\
=\cos (\psi-\sigma) \sin \varphi \sin \gamma \sin \theta \\
\quad+\sin (\psi-\sigma) \cos \gamma \sin \theta+\cos \varphi \sin \gamma \cos \theta, \\
C_{1}=x \cos \theta \cos \sigma+\left(y+R_{e}\right) \sin \theta-z \cos \theta \sin \sigma, \\
C_{2}=-x \sin \theta \cos \sigma+\left(y+R_{e}\right) \cos \theta+z \sin \theta \sin \sigma, \\
\quad C_{3}=x \sin \sigma+z \cos \sigma, \\
C_{4}=\sin \alpha \cos \gamma_{V}+\cos \alpha \sin \beta \sin \gamma_{V}, \\
C_{5}=\sin \alpha \sin \gamma_{V}-\cos \alpha \sin \beta \cos \gamma_{V},
\end{gathered}
$$

where $\alpha, \beta$, and $\gamma_{V}$ are the angle-of-attack, sideslip angle, and bank angle, respectively; $\varphi, \psi$, and $\gamma$ are the pitch, yaw, and rolling angles, respectively; $v, \theta$, and $\sigma$ are the flight velocity, flight path angle, and trajectory angle, respectively; $\omega_{x}, \omega_{y}$, and $\omega_{z}$ are pitch, yaw, and roll rates, respectively; $x, y, z$ are the three position coordinates in the ground coordinate frame; $R_{e}, r$ are radial distance from the Earth's center and the mean radius of the spherical Earth, respectively; $M_{x}, M_{y}$, and $M_{z}$ are control moments including roll, yaw, and pitch control moments; $L, N$, and $T$ are the lift, side, and thrust forces, respectively; $J_{x}, J_{y}$, and $J_{z}$ are the main moments of inertia of the three axes, respectively. 
Remark 1. For the convenience of control system design, $[\varphi, \psi, \gamma]^{\mathrm{T}}$ is selected as the state variables. In view of the aim of attitude control system design for $\mathrm{HV}$ which is to track the guidance commands $\left[\alpha_{c}, \beta_{c}, \gamma_{V_{c}}\right]^{\mathrm{T}}$ precisely, it is necessary to transform $\left[\alpha_{c}, \beta_{c}, \gamma_{V_{c}}\right]^{\mathrm{T}}$ into $\left[\varphi_{c}, \psi_{c}, \gamma_{c}\right]^{\mathrm{T}}$ by means of (3) which is obtained by the coordinate system transformation.

\section{Predictive Sliding Mode Control Based on Fuzzy Disturbance Observer}

3.1. Predictive Sliding Mode Control for Systems with Uncertainties and Disturbances. To develop the predictive sliding mode control, we consider the following MIMO uncertain affine nonlinear system with external disturbances:

$$
\begin{aligned}
& \dot{\mathbf{x}}=\mathbf{f}(\mathbf{x})+\Delta \mathbf{f}(\mathbf{x})+(\mathbf{g}(\mathbf{x})+\Delta \mathbf{g}(\mathbf{x})) \mathbf{u}+\mathbf{d}, \\
& \mathbf{y}=\mathbf{h}(\mathbf{x})
\end{aligned}
$$

where $\mathbf{x} \in \mathbf{R}^{n}$ is the state vector of the uncertain nonlinear system; $\mathbf{u} \in \mathbf{R}^{m}$ is the control input vector; $\mathbf{y} \in \mathbf{R}^{m}$ is the output vector of the uncertain nonlinear system; $\mathbf{f}(\mathbf{x}) \in \mathbf{R}^{n}$ and $\mathbf{g}(\mathbf{x}) \in \mathbf{R}^{n \times m}$ are the given function vector and control gain matrix, respectively; $\Delta \mathbf{f}(\mathbf{x})$ and $\Delta \mathbf{g}(\mathbf{x})$ are the internal uncertainty and modeling error; and $\mathbf{d}$ is the external timevarying disturbance.

Define

$$
\mathbf{D}(\mathbf{x}, \mathbf{u}, \mathbf{d})=\Delta \mathbf{f}(\mathbf{x})+\Delta \mathbf{g}(\mathbf{x}) \mathbf{u}+\mathbf{d},
$$

where $\mathbf{D}(\mathbf{x}, \mathbf{u}, \mathbf{d})$ denotes the system uncertainties and external disturbances.

Without loss of generality, the equilibrium of uncertain nonlinear system (5) is supposed to be $\mathbf{x}_{0}=0$. In accordance with the Lie derivative operation [27] in differential geometry theory, the vector relative degree of MIMO system can be defined as follows.

Definition 2 (see [27]). The vector relative degree of system (5) is $\left\{\rho_{1}, \rho_{2}, \ldots, \rho_{m}\right\}$ at $\mathbf{x}_{0}$ under the following conditions, where $\rho_{1}, \rho_{2}, \ldots, \rho_{m}$ denote the relative degree of each channel, respectively.

(1) For all $\mathbf{x}$ in a neighborhood of $\mathbf{x}_{0}, L_{\mathbf{g}_{j}} L_{\mathbf{f}}^{k} h_{i}(\mathbf{x})=0(1 \leq$ $\left.i, j \leq m, 0<k<\rho_{i}\right)$.

(2) The following $m \times m$ matrix is nonsingular at $\mathbf{x}_{0}=0$ :

\section{$\mathbf{A}(\mathbf{x})$}

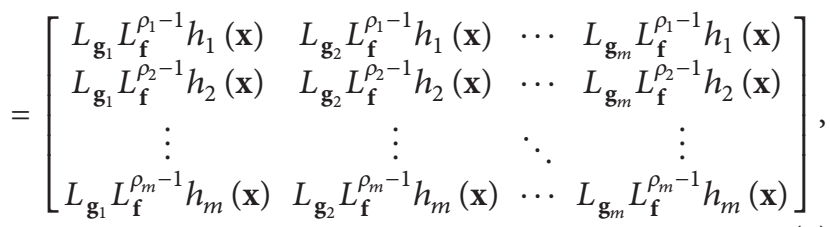

where $\mathbf{g}_{j}$ is the $j$ th row vector of $\mathbf{g}(\mathbf{x})$ and $h_{i}(\mathbf{x})$ is the $i$ th output of system (5). Similarly, the disturbance relative degree at $\mathbf{x}_{0}$ can be defined as $\left\{\tau_{1}, \tau_{2}, \ldots, \tau_{m}\right\}$.
To facilitate the process of control system design, the following reasonable assumptions are required before developing predictive sliding mode control of the uncertain MIMO nonlinear system (5).

Assumption 3. $\mathbf{f}(\mathbf{x})$ and $\mathbf{h}(\mathbf{x})$ are continuously differentiable, and $\mathbf{g}(\mathbf{x})$ is continuous.

Assumption 4. All states are available; moreover, the output and reference signals are also continuously differentiable.

Assumption 5. The zero dynamics are stable.

Assumption 6. The vector relative degree is $\left\{\rho_{1}, \rho_{2}, \ldots, \rho_{m}\right\}$, and the disturbance relative degree of composite disturbances is $\left\{\tau_{1}, \tau_{2}, \ldots, \tau_{m}\right\}$, where $\tau_{i} \geq \rho_{i}(i=1, \ldots, m)$.

According to Assumption 6, we can obtain

$$
\begin{aligned}
y_{i}^{\left(\rho_{i}\right)}= & L_{f}^{\rho_{i}} h_{i}(\mathbf{x})+\left(L_{g} L_{f}^{\rho_{i}-1} h_{i}(\mathbf{x})\right) \mathbf{u} \\
& +L_{D} L_{f}^{\rho_{i}-1} h_{i}(\mathbf{x}) \quad(i=1, \ldots, m) .
\end{aligned}
$$

Associate the $m$ equations; the nonlinear system (5) can be written as

$$
\mathbf{y}^{(\rho)}=\boldsymbol{\alpha}(\mathbf{x})+\boldsymbol{\beta}(\mathbf{x}) \mathbf{u}+\Delta(\mathbf{x}, \mathbf{u}, \mathbf{D}),
$$

where

$$
\begin{aligned}
\boldsymbol{\alpha}(\mathbf{x}) & =\left[\alpha_{1}, \alpha_{2} \cdots \alpha_{m}\right]^{\mathrm{T}} \in \mathbf{R}^{m} \\
\boldsymbol{\beta}(\mathbf{x}) & =\left[\beta_{1}, \beta_{2} \cdots \beta_{m}\right]^{\mathrm{T}} \in \mathbf{R}^{m \times m} \\
\Delta(\mathbf{x}, \mathbf{u}, \mathbf{D}) & =\left[\delta_{1}, \delta_{2} \cdots \delta_{m}\right]^{\mathrm{T}} \in \mathbf{R}^{m},
\end{aligned}
$$

where $\alpha_{i}=L_{f}^{\rho_{i}} h_{i}(\mathbf{x}), \beta_{i}=L_{g} L_{f}^{\rho_{i}-1} h_{i}(\mathbf{x}), \delta_{i}=L_{D} L_{f}^{\rho_{i}-1} h_{i}(\mathbf{x})(i=$ $1, \ldots, m)$, and $\Delta(\mathbf{x}, \mathbf{u}, \mathbf{D})$ is related to the composite disturbances.

Furthermore, the sliding surface vector is defined as

$$
\mathbf{s}(t)=\left[s_{1}, s_{2} \cdots s_{m}\right]^{\mathrm{T}} \in \mathbf{R}^{m},
$$

where each sliding surface is the combination of the tracking error and its higher order derivatives, which can be depicted as

$$
s_{i}=e_{i}^{\left[\rho_{i}-1\right]}+k_{i, \rho_{i}-1} e_{i}^{\left[\rho_{i}-2\right]}+\cdots+k_{i, 0} e_{i},
$$

where $e_{i}=y_{i}-y_{c i}(i=1, \ldots, m)$ and $k_{i, j}(j=$ $\left.0,1, \ldots, \rho_{i}-1\right)$ must make the polynomial (12) Hurwitz stable. Differentiating (12), we have

$$
\begin{aligned}
\dot{s}_{i}= & e_{i}^{\left[\rho_{i}\right]}+k_{i, \rho_{i}-1} e_{i}^{\left[\rho_{i}-1\right]}+\cdots+k_{i, 0} \dot{e}_{i} \\
= & y_{i}^{\left[\rho_{i}\right]}-y_{c i}^{\left[\rho_{i}\right]}+k_{i, \rho_{i}-1} e_{i}^{\left[\rho_{i}-1\right]} \\
& +\cdots+k_{i, 0} \dot{e}_{i} \quad(i=1, \ldots, m) .
\end{aligned}
$$

For the sake of simplified notation, define $z_{i}$ as

$$
z_{i}=k_{i, \rho_{i}-1} e_{i}^{\left[\rho_{i}-1\right]}+\cdots+k_{i, 0} \dot{e}_{i} \quad(i=1, \ldots, m) .
$$


Then the vector $\mathbf{z}$ can be written as

$$
\mathbf{z}=\left[z_{1}, z_{2} \cdots z_{m}\right]^{\mathrm{T}} \in \mathbf{R}^{m} .
$$

Consequently, differentiating the sliding surface vector, we obtain

$$
\dot{\mathbf{s}}(t)=\mathbf{y}^{(\rho)}-\mathbf{y}_{\mathbf{c}}^{(\rho)}+\mathbf{z},
$$

where $\mathbf{y}_{\mathbf{c}}^{(\boldsymbol{\rho})}=\left[y_{c 1}^{\left(\rho_{1}\right)}, y_{c 2}^{\left(\rho_{2}\right)} \cdots y_{c m}^{\left(\rho_{m}\right)}\right]^{\mathrm{T}} \in \mathbf{R}^{m}$.

Within the moving time frame, the sliding surface $\widehat{\mathbf{s}}(t+T)$ at the time $T$ is approximately predicted by

$$
\widehat{\mathbf{s}}(t+T)=\mathbf{s}(t)+T \dot{\mathbf{s}}(t) .
$$

For the derivation of the control law, the recedinghorizon performance index at the time $T$ is given by

$$
J(\mathbf{x}, \mathbf{u}, t)=\frac{1}{2} \widehat{\mathbf{s}}^{\mathrm{T}}(t+T) \widehat{\mathbf{s}}(t+T) .
$$

The necessary condition for the optimal control to minimize (18) with respect to $\mathbf{u}$ is given by

$$
\frac{\partial J}{\partial \mathbf{u}}=0 .
$$

According to (19), substitute (17) into (18); then, the predictive sliding mode control law can be proposed as

$$
\begin{aligned}
\mathbf{u}= & -T^{-1} \boldsymbol{\beta}(x)^{-1} \\
& \cdot\left(\mathbf{s}(t)+T\left(\boldsymbol{\alpha}(x)+\Delta(\mathbf{x}, \mathbf{u}, \mathbf{D})-\mathbf{y}_{\mathbf{c}}^{(\boldsymbol{\rho})}+\mathbf{z}\right)\right) .
\end{aligned}
$$

Remark 7. If $\mathbf{D}=0$, the control law given by (20) is the nominal predictive sliding mode control law. If $\mathbf{D} \neq 0$, $\Delta(\mathbf{x}, \mathbf{u}, \mathbf{D})$ cannot be directly obtained due to the immeasurable unknown composite disturbances $\mathbf{D}$. The performance of flight control system may become worse, even unstable, when D is big enough. To handle this problem, a fuzzy observer would be designed to estimate the composite disturbances.

Remark 8. For the convenience of notation, $\Delta(\mathbf{x}, \mathbf{u}, \mathbf{D})$ would be denoted by $\Delta(\mathbf{x})$ in the rest of this paper.

\subsection{Fuzzy Disturbance Observer}

3.2.1. Fuzzy Logic System. The fuzzy inference engine adopts the fuzzy if-then rules to perform a mapping from an input linguistic vector $\mathbf{X}=\left[X_{1}, X_{2}, \ldots, X_{n}\right]^{\mathrm{T}} \in \mathbf{R}^{n}$ to an output variable $Y \in \mathbf{R}$. The $i$ th fuzzy rule can be expressed as [19]

$$
R^{(i)}: \text { If } X_{1} \text { is } A_{1}^{i}, \ldots, \quad X_{n} \text { is } A_{n}^{i} \text {, then } Y \text { is } Y^{i},
$$

where $A_{1}^{i}, \ldots, A_{n}^{i}$ are fuzzy sets characterized by fuzzy membership functions and $Y^{i}$ is a singleton number.

By adopting a center-average and singleton fuzzifier and the product inference, the output of the fuzzy system can be written as

$$
Y(\mathbf{X})=\frac{\sum_{i=1}^{l} Y^{i}\left(\prod_{j=1}^{n} \mu_{A_{j}^{i}}\left(X_{j}\right)\right)}{\sum_{i=1}^{l}\left(\prod_{j=1}^{n} \mu_{A_{j}^{i}}\left(X_{j}\right)\right)}=\widehat{\boldsymbol{\theta}}^{\mathrm{T}} \boldsymbol{\vartheta}(\mathbf{X}),
$$

where $l$ is the number of fuzzy rules, $\mu_{A_{j}^{i}}\left(X_{j}\right)$ is the membership function value of fuzzy variable $X_{j}(j=1,2, \ldots, n)$, $\widehat{\boldsymbol{\theta}}=\left[Y^{1}, Y^{2}, \ldots, Y^{l}\right]^{\mathrm{T}}$ is an adjustable parameter vector, and $\boldsymbol{\vartheta}(\mathbf{x})=\left[\vartheta^{1}, \vartheta^{2}, \ldots, \vartheta^{l}\right]^{\mathrm{T}}$ is a fuzzy basis function vector. $\vartheta^{i}$ can be expressed as

$$
\vartheta^{i}=\frac{\prod_{j=1}^{n} \mu_{A_{j}^{i}}\left(X_{j}\right)}{\sum_{i=1}^{l}\left(\prod_{j=1}^{n} \mu_{A_{j}^{i}}\left(X_{j}\right)\right)} .
$$

Lemma 9 (see [28]). For any given real continuous function $\mathbf{g}(\mathbf{x})$ on the compact set $\mathbf{U} \in \mathbf{R}^{n}$ and arbitrary $\varepsilon>0$, there exists a fuzzy system $p(\mathbf{X})$ presented as (22) such that

$$
\sup _{x \in \mathbf{U}}|p(\mathbf{X})-Y(\mathbf{X})|<\varepsilon .
$$

According to Lemma 9, a fuzzy system can be designed to approximate the composite disturbances $\Delta(\mathbf{x})$ by adjusting the parameter vector $\widehat{\boldsymbol{\theta}}$ online. The designed fuzzy system can be written as

$$
\widehat{\Delta}\left(\mathbf{x} \mid \widehat{\boldsymbol{\theta}}_{\Delta}^{\mathrm{T}}\right)=\left[\widehat{\delta}_{1}, \widehat{\delta}_{2} \cdots \widehat{\delta}_{m}\right]^{\mathrm{T}}=\widehat{\boldsymbol{\theta}}_{\Delta}^{\mathrm{T}} \boldsymbol{\vartheta}_{\Delta}(\mathbf{x}),
$$

where

$$
\begin{aligned}
\widehat{\boldsymbol{\theta}}_{\Delta}^{\mathrm{T}} & =\operatorname{diag}\left\{\widehat{\boldsymbol{\theta}}_{1}^{\mathrm{T}}, \widehat{\boldsymbol{\theta}}_{2}^{\mathrm{T}}, \ldots, \widehat{\boldsymbol{\theta}}_{m}^{\mathrm{T}}\right\}, \\
\widehat{\boldsymbol{\theta}}_{i}^{\mathrm{T}} & =\left(Y_{i}^{1}, Y_{i}^{2}, \ldots, Y_{i}^{l}\right)^{\mathrm{T}}, \\
\boldsymbol{\vartheta}_{\Delta}(\mathbf{x}) & =\left[\boldsymbol{\vartheta}_{1}(\mathbf{x})^{\mathrm{T}}, \boldsymbol{\vartheta}_{2}(\mathbf{x})^{\mathrm{T}}, \ldots, \boldsymbol{\vartheta}_{m}(\mathbf{x})^{\mathrm{T}}\right]^{\mathrm{T}}, \\
\boldsymbol{\vartheta}(\mathbf{x}) & =\left[\vartheta_{i}^{1}, \vartheta_{i}^{2}, \ldots, \vartheta_{i}^{l}\right]^{\mathrm{T}} .
\end{aligned}
$$

Let $\mathbf{x}$ belong to a compact set $\boldsymbol{\Omega}_{\mathbf{x}}$; the optimal parameter vector $\boldsymbol{\theta}_{\Delta}^{* \mathrm{~T}}$ can be defined as

$$
\boldsymbol{\theta}_{\Delta}^{* \mathrm{~T}}=\arg \min _{\widehat{\boldsymbol{\theta}}_{\Delta}^{\mathrm{T}}}\left\{\sup _{\mathbf{x} \in \boldsymbol{\Omega}_{\mathbf{x}}}\left\|\Delta(\mathbf{x})-\widehat{\Delta}\left(\mathbf{x} \mid \widehat{\boldsymbol{\theta}}_{\Delta}^{\mathrm{T}}\right)\right\|\right\},
$$

where the optimal parameter matrix $\boldsymbol{\theta}_{\Delta}^{* \mathrm{~T}}$ lies in a convex region given by

$$
\mathbf{M}_{\boldsymbol{\theta}}=\left\{\widehat{\boldsymbol{\theta}}_{\Delta} \mid\left\|\widehat{\boldsymbol{\theta}}_{\Delta}\right\| \leq m_{\theta}\right\},
$$

where $\|\cdot\|$ is the Frobenius norm and $m_{\theta}$ is a design parameter with $m_{\theta}>0$.

Consequently, the composite disturbances $\Delta(\mathbf{x})$ can be written as

$$
\Delta(\mathbf{x})=\boldsymbol{\theta}_{\Delta}^{* \mathrm{~T}} \boldsymbol{\vartheta}_{\Delta}(\mathbf{x})+\boldsymbol{\varepsilon},
$$

where $\boldsymbol{\varepsilon}$ is the smallest approximation error of the fuzzy system. Apparently, the norm of $\boldsymbol{\theta}_{\Delta}^{* \mathrm{~T}}$ is bounded with

$$
\|\boldsymbol{\varepsilon}\| \leq \bar{\varepsilon},
$$

where $\bar{\varepsilon}$ is the upper bound of the approximation error $\boldsymbol{\varepsilon}$. 
Through adjusting the parameter vector $\widehat{\boldsymbol{\theta}}$ online, the composite disturbances $\Delta(\mathbf{x})$ can be approximated by the fuzzy system; hence, the performance of the control system with composite disturbances can be improved. As the performance of the control system is closely related to the selected fuzzy system, the control precision will be reduced when the approximation ability of the selected fuzzy system is dissatisfactory. In order to improve the approximation ability, a hyperbolic tangent function is integrated with the fuzzy disturbance observer to compensate for the approximate error.

3.2.2. Fuzzy Disturbance Observer. Consider the following dynamic system:

$$
\dot{\boldsymbol{\mu}}=-\chi \boldsymbol{\mu}+p\left(\mathbf{x}, \mathbf{u}, \widehat{\boldsymbol{\theta}}_{\Delta}\right),
$$

where $p\left(\mathbf{x}, \mathbf{u}, \widehat{\boldsymbol{\theta}}_{\Delta}\right)=\chi \mathbf{y}^{(\rho-1)}+\boldsymbol{\alpha}(\mathbf{x})+\boldsymbol{\beta}(\mathbf{x}) \mathbf{u}+\widehat{\Delta}\left(\mathbf{x} \mid \widehat{\boldsymbol{\theta}}_{\Delta}^{\mathrm{T}}\right), \chi>0$ is a design parameter, and $\widehat{\Delta}\left(\mathbf{x} \mid \widehat{\boldsymbol{\theta}}_{\Delta}^{\mathrm{T}}\right)=\widehat{\boldsymbol{\theta}}_{\Delta}^{\mathrm{T}} \boldsymbol{\vartheta}_{\Delta}(\mathbf{x})$ is utilized to compensate for the composite disturbances.

Define the disturbance observation error $\zeta=\mathbf{y}^{(\rho-1)}-\boldsymbol{\mu}$; invoking (9) and (31) yields

$$
\begin{aligned}
\dot{\zeta}= & \boldsymbol{\alpha}(\mathbf{x})+\boldsymbol{\beta}(\mathbf{x}) \mathbf{u}+\Delta(\mathbf{x})+\sigma \boldsymbol{\mu}-\boldsymbol{\sigma} \mathbf{y}^{(\boldsymbol{\rho}-1)}-\boldsymbol{\alpha}(\mathbf{x}) \\
& -\boldsymbol{\beta}(\mathbf{x}) \mathbf{u}-\widehat{\Delta}\left(\mathbf{x} \mid \widehat{\boldsymbol{\theta}}_{\Delta}^{\mathrm{T}}\right)=-\sigma \zeta+\widetilde{\boldsymbol{\theta}}_{\Delta}^{\mathrm{T}} \boldsymbol{\vartheta}_{\Delta}(\mathbf{x})+\boldsymbol{\varepsilon},
\end{aligned}
$$

where $\widetilde{\boldsymbol{\theta}}_{\Delta}=\boldsymbol{\theta}_{\Delta}^{*}-\widehat{\boldsymbol{\theta}}_{\Delta}$ is the adjustable parameter error vector.

Then, the FDO-PSMC is proposed as

$$
\begin{aligned}
\mathbf{u}= & -T^{-1} \boldsymbol{\beta}(x)^{-1} \\
& \cdot\left(\mathbf{s}(t)+T\left(\boldsymbol{\alpha}(x)+\widehat{\boldsymbol{\theta}}_{\Delta}^{\mathrm{T}} \boldsymbol{\vartheta}_{\Delta}(\mathbf{x})-\mathbf{y}_{\mathbf{c}}^{(\boldsymbol{\rho})}+\mathbf{z}\right)\right) .
\end{aligned}
$$

Invoking (16) and (33), we have

$$
\begin{aligned}
\dot{\mathbf{s}} & =\mathbf{y}^{(\rho)}-\mathbf{y}_{\mathbf{c}}^{(\rho)}+\mathbf{z}=\boldsymbol{\alpha}(\mathbf{x})+\boldsymbol{\beta}(\mathbf{x}) \mathbf{u}+\Delta(\mathbf{x})-\mathbf{y}_{\mathbf{c}}^{(\rho)}+\mathbf{z} \\
& =-T^{-1} \mathbf{s}+\widetilde{\boldsymbol{\theta}}_{\Delta}^{\mathrm{T}} \boldsymbol{\vartheta}_{\Delta}(\mathbf{x})+\boldsymbol{\varepsilon} .
\end{aligned}
$$

Invoking (32) and (34), the augmented system is obtained as

$$
\dot{\boldsymbol{\zeta}}=\mathbf{A} \boldsymbol{\varsigma}+\mathbf{B}\left(\widetilde{\boldsymbol{\theta}}_{\Delta}^{\mathrm{T}} \boldsymbol{\vartheta}_{\Delta}(\mathbf{x})+\boldsymbol{\varepsilon}\right),
$$

where $=\left(\boldsymbol{\zeta}^{\mathrm{T}}, \mathbf{s}^{\mathrm{T}}\right)^{\mathrm{T}}, \mathbf{A}=\operatorname{diag}\left(-\chi \mathbf{I}_{m},-T^{-1} \mathbf{I}_{m}\right)$, and $\mathbf{B}=$ $\left(\mathbf{I}_{m}, \mathbf{I}_{m}\right)^{\mathrm{T}}$.

Theorem 10. Assume that the disturbance of (9) is monitored by the system (32), and the system (9) is controlled by (33). If the adjustable parameter vector of FDO is tuned by (36), then the augmented error $\boldsymbol{\varsigma}$ is uniformly ultimately bounded within an arbitrarily small region:

$$
\begin{aligned}
\dot{\hat{\boldsymbol{\theta}}}_{\Delta} & =\operatorname{Proj}\left[\lambda \boldsymbol{\vartheta}_{\Delta}(\mathbf{x}) \boldsymbol{\varsigma}^{\mathrm{T}} \mathbf{B}\right] \\
& =\lambda \boldsymbol{\vartheta}_{\Delta}(\mathbf{x}) \boldsymbol{\varsigma}^{\mathrm{T}}-I_{\theta} \lambda \frac{\boldsymbol{\varsigma}^{\mathrm{T}} \mathbf{B} \widehat{\boldsymbol{\theta}}_{\Delta}^{\mathrm{T}} \boldsymbol{\vartheta}_{\Delta}(\mathbf{x})}{\left\|\widehat{\boldsymbol{\theta}}_{\Delta}\right\|^{2}} \widehat{\boldsymbol{\theta}}_{\Delta},
\end{aligned}
$$

where

$I_{\theta}$

$$
= \begin{cases}0, & \text { if }\left\|\widehat{\boldsymbol{\theta}}_{\Delta}\right\|<m_{\theta}, \text { or }\left\|\widehat{\boldsymbol{\theta}}_{\Delta}\right\|=m_{\theta}, \boldsymbol{\varsigma}{ }^{\mathrm{T}} \mathbf{B} \widehat{\boldsymbol{\theta}}_{\Delta}^{\mathrm{T}} \boldsymbol{\vartheta}_{\Delta}(\mathbf{x}) \leq 0 \\ 1, & \text { if }\left\|\widehat{\boldsymbol{\theta}}_{\Delta}\right\|=m_{\theta}, \boldsymbol{c}{ }^{\mathrm{T}} \mathbf{B} \widehat{\boldsymbol{\theta}}_{\Delta}^{\mathrm{T}} \boldsymbol{\vartheta}_{\Delta}(\mathbf{x})>0 .\end{cases}
$$

Proof. Consider the Lyapunov function candidate:

$$
V=\frac{1}{2} \boldsymbol{c}^{\mathrm{T}} \boldsymbol{\varsigma}+\frac{1}{2 \lambda} \widetilde{\boldsymbol{\theta}}_{\Delta}^{\mathrm{T}} \widetilde{\boldsymbol{\theta}}_{\Delta} .
$$

Invoking (35) and (36), the time derivative of $V$ is

$$
\begin{aligned}
\dot{V}= & \boldsymbol{\varsigma}^{\mathrm{T}} \dot{\boldsymbol{\xi}}+\frac{1}{\lambda} \widetilde{\boldsymbol{\theta}}_{\Delta}^{\mathrm{T}} \dot{\tilde{\boldsymbol{\theta}}}_{\Delta}=\boldsymbol{\zeta}^{\mathrm{T}} \dot{\boldsymbol{\zeta}}+\mathbf{s}^{\mathrm{T}} \dot{\boldsymbol{s}}+\frac{1}{\lambda} \widetilde{\boldsymbol{\theta}}_{\Delta}^{\mathrm{T}} \dot{\tilde{\boldsymbol{\theta}}}_{\Delta} \\
= & \boldsymbol{\zeta}^{\mathrm{T}}\left(-\chi \zeta+\widetilde{\boldsymbol{\theta}}_{\Delta}^{\mathrm{T}} \boldsymbol{\vartheta}_{\Delta}(\mathbf{x})+\boldsymbol{\varepsilon}\right)+\mathbf{s}^{\mathrm{T}}\left(-T^{-1} \mathbf{s}+\widetilde{\boldsymbol{\theta}}_{\Delta}^{\mathrm{T}} \boldsymbol{\vartheta}_{\Delta}(\mathbf{x})+\boldsymbol{\varepsilon}\right) \\
& -\frac{1}{\lambda} \widetilde{\boldsymbol{\theta}}_{\Delta}^{\mathrm{T}} \operatorname{Proj}\left[\lambda \boldsymbol{\varphi}_{\Delta}(\mathbf{x}) \boldsymbol{\varsigma}^{\mathrm{T}} \mathbf{B}\right]+\frac{1}{\boldsymbol{\eta}} \widetilde{\boldsymbol{\vartheta}} \dot{\tilde{\boldsymbol{\vartheta}}} \\
= & \boldsymbol{\zeta}^{\mathrm{T}}\left(-\chi \zeta+\widetilde{\boldsymbol{\theta}}_{\Delta}^{\mathrm{T}} \boldsymbol{\vartheta}_{\Delta}(\mathbf{x})+\boldsymbol{\varepsilon}\right)+\mathbf{s}^{\mathrm{T}}\left(-T^{-1} \mathbf{s}+\widetilde{\boldsymbol{\theta}}_{\Delta}^{\mathrm{T}} \boldsymbol{\vartheta}_{\Delta}(\mathbf{x})+\boldsymbol{\varepsilon}\right) \\
& -\widetilde{\boldsymbol{\theta}}_{\Delta}^{\mathrm{T}} \boldsymbol{\vartheta}_{\Delta}(\mathbf{x}) \boldsymbol{\varsigma}^{\mathrm{T}}+\widetilde{\boldsymbol{\theta}}_{\Delta}^{\mathrm{T}} I_{\theta} \frac{\boldsymbol{\varsigma}^{\mathrm{T}} \mathbf{B} \widehat{\boldsymbol{\theta}}_{\Delta}^{\mathrm{T}} \boldsymbol{\vartheta}_{\Delta}(\mathbf{x})}{\left\|\widehat{\boldsymbol{\theta}}_{\Delta}\right\|^{2}} \widehat{\boldsymbol{\theta}}_{\Delta} .
\end{aligned}
$$

Considering the fact

$$
\begin{aligned}
\widetilde{\boldsymbol{\theta}}_{\Delta}^{\mathrm{T}} \widehat{\boldsymbol{\theta}}_{\Delta} & =\boldsymbol{\theta}_{\Delta}^{* \mathrm{~T}} \widehat{\boldsymbol{\theta}}_{\Delta}-\widehat{\boldsymbol{\theta}}_{\Delta}^{\mathrm{T}} \widehat{\boldsymbol{\theta}}_{\Delta} \\
& \leq \frac{1}{2}\left(\left\|\boldsymbol{\theta}_{\Delta}^{*}\right\|^{2}+\left\|\hat{\boldsymbol{\theta}}_{\Delta}\right\|^{2}\right)-\left\|\widehat{\boldsymbol{\theta}}_{\Delta}\right\|^{2} \\
& \leq \frac{1}{2}\left(\left\|\boldsymbol{\theta}_{\Delta}^{*}\right\|^{2}-m_{\theta}^{2}\right) \\
& \leq 0 \quad\left(\text { when }\left\|\widehat{\boldsymbol{\theta}}_{\Delta}\right\|=m_{\theta}\right)
\end{aligned}
$$

and invoking (37), we have

$$
\begin{aligned}
& \tilde{\boldsymbol{\theta}}_{\Delta}^{\mathrm{T}} I_{\theta} \frac{\boldsymbol{c}^{\mathrm{T}} \mathbf{B} \widehat{\boldsymbol{\theta}}_{\Delta}^{\mathrm{T}} \boldsymbol{\vartheta}_{\Delta}(\mathbf{x})}{\left\|\widehat{\boldsymbol{\theta}}_{\Delta}\right\|^{2}} \widehat{\boldsymbol{\theta}}_{\Delta}=0 \\
& \text { or } \tilde{\boldsymbol{\theta}}_{\Delta}^{\mathrm{T}} I_{\theta} \frac{\boldsymbol{c}^{\mathrm{T}} \mathbf{B} \widehat{\boldsymbol{\theta}}_{\Delta}^{\mathrm{T}} \boldsymbol{\vartheta}_{\Delta}(\mathbf{x})}{\left\|\widehat{\boldsymbol{\theta}}_{\Delta}\right\|^{2}} \widehat{\boldsymbol{\theta}}_{\Delta}<0 .
\end{aligned}
$$

Consequently, we obtain

$$
\begin{aligned}
\dot{V} \leq & \zeta^{\mathrm{T}}\left(-\chi \zeta+\tilde{\boldsymbol{\theta}}_{\Delta}^{\mathrm{T}} \boldsymbol{\varphi}_{\Delta}(\mathbf{x})+\boldsymbol{\varepsilon}\right) \\
& +\mathbf{s}^{\mathrm{T}}\left(-T^{-1} \mathbf{s}+\tilde{\boldsymbol{\theta}}_{\Delta}^{\mathrm{T}} \boldsymbol{\varphi}_{\Delta}(\mathbf{x})+\boldsymbol{\varepsilon}\right)-\widetilde{\boldsymbol{\theta}}_{\Delta}^{\mathrm{T}} \boldsymbol{\varphi}_{\Delta}(\mathbf{x}) \boldsymbol{\varsigma}^{\mathrm{T}} \\
\leq & \boldsymbol{\zeta}^{\mathrm{T}}\left(-\chi \zeta+\tilde{\boldsymbol{\theta}}_{\Delta}^{\mathrm{T}} \boldsymbol{\varphi}_{\Delta}(\mathbf{x})+\boldsymbol{\varepsilon}\right)+\mathbf{s}^{\mathrm{T}}\left(-T^{-1} \mathbf{s}+\widetilde{\boldsymbol{\theta}}_{\Delta}^{\mathrm{T}} \boldsymbol{\varphi}_{\Delta}(\mathbf{x})+\boldsymbol{\varepsilon}\right) \\
& -\tilde{\boldsymbol{\theta}}_{\Delta}^{\mathrm{T}} \boldsymbol{\varphi}_{\Delta}(\mathbf{x}) \boldsymbol{\zeta}-\tilde{\boldsymbol{\theta}}_{\Delta}^{\mathrm{T}} \boldsymbol{\varphi}_{\Delta}(\mathbf{x}) \mathbf{s} \\
\leq & \boldsymbol{\zeta}^{\mathrm{T}}(-\chi \zeta+\boldsymbol{\varepsilon})+\mathbf{s}^{\mathrm{T}}\left(-T^{-1} \mathbf{s}+\boldsymbol{\varepsilon}\right)
\end{aligned}
$$




$$
\begin{aligned}
& \leq-\chi\|\zeta\|^{2}+\boldsymbol{\zeta}^{\mathrm{T}} \boldsymbol{\varepsilon}-T^{-1}\|\mathbf{s}\|^{2}+\mathbf{s}^{\mathrm{T}} \boldsymbol{\varepsilon} \\
& \leq-\frac{\chi}{2}\|\boldsymbol{\zeta}\|^{2}+\frac{1}{2 \chi}\|\boldsymbol{\varepsilon}\|^{2}-\frac{1}{2 T}\|\mathbf{s}\|^{2}+\frac{T}{2}\|\boldsymbol{\varepsilon}\|^{2} .
\end{aligned}
$$

If $\|\zeta\|>\bar{\varepsilon} / \chi$ or $\|\mathbf{s}\|>T \bar{\varepsilon}, \dot{V}<0$. Therefore, the augmented error $\varsigma$ is uniformly ultimately bounded.

Remark 11. In order to make sure that the FDO $\widehat{\Delta}\left(\mathbf{x} \mid \widehat{\boldsymbol{\theta}}_{\Delta}^{\mathrm{T}}\right)$ outputs zero signal in case of the composite disturbances $\mathbf{D}=$ 0 , the consequent parameters should be set to be 0 :

$$
\widehat{\boldsymbol{\theta}}_{\Delta}(0)=0 \text {. }
$$

Remark 12. A projection operator (36) is used in the tuning method of the adjustable parameter vector; then, $\left\|\widehat{\boldsymbol{\theta}}_{\Delta}\right\|$ can be guaranteed to be bounded [29].

Remark 13. The adjustable parameter vector of the FDO (36) includes the observation error $\zeta$ and the sliding surface $\mathbf{s}$. In view of the fact that the sliding surface $s$ which is the linearization combination of the tracking error $\mathbf{e}$ is Hurwitz stable, the observation error $\zeta$ and tracking error e are both uniformly ultimately bounded, which ensures stability of the control system.

\subsection{Improved Fuzzy Disturbance Observer}

Lemma 14 (see [30]). For all $c>0$ and $\mathbf{w} \in R^{m}$, the following inequality holds:

$$
0<\|\mathbf{w}\|-\mathbf{w}^{\mathrm{T}} \tanh \left(\frac{\mathbf{w}}{c}\right) \leq m \kappa c
$$

where $\kappa$ is a constant such that $\kappa=\mathrm{e}^{-(\kappa+1)}$; that is, $\kappa=0.2785$.

The hyperbolic tangent function is incorporated to compensate for the approximate error and improve the precision of FDO. Consider the following dynamic system:

$$
\dot{\boldsymbol{\mu}}=-\chi \boldsymbol{\mu}+p\left(\mathbf{x}, \mathbf{u}, \widehat{\boldsymbol{\theta}}_{\Delta}\right),
$$

where $p\left(\mathbf{x}, \mathbf{u}, \widehat{\boldsymbol{\theta}}_{\Delta}\right)=\sigma \mathbf{y}^{(\rho-1)}+\boldsymbol{\alpha}(\mathbf{x})+\boldsymbol{\beta}(\mathbf{x}) \mathbf{u}+\widehat{\Delta}(\mathbf{x}$ $\left.\widehat{\boldsymbol{\theta}}_{\Delta}^{\mathrm{T}}\right)-\widehat{v} \tanh (\boldsymbol{\zeta} / \boldsymbol{c}), \chi>0$ is a design parameter, and $\widehat{\Delta}(\mathbf{x}$ $\left.\widehat{\boldsymbol{\theta}}_{\Delta}^{\mathrm{T}}\right)=\widehat{\boldsymbol{\theta}}_{\Delta}^{\mathrm{T}} \boldsymbol{\vartheta}_{\Delta}(\mathbf{x})$ is utilized to compensate for the composite disturbances.

Define the disturbance observation error $\boldsymbol{\zeta}=\mathbf{y}^{(\rho-1)}-\boldsymbol{\mu}$; invoking (9) and (45) yields

$$
\begin{aligned}
\dot{\boldsymbol{\zeta}}= & \boldsymbol{\alpha}(\mathbf{x})+\boldsymbol{\beta}(\mathbf{x}) \mathbf{u}+\Delta(\mathbf{x})+\sigma \boldsymbol{\mu}-\boldsymbol{\sigma} \mathbf{y}^{(\rho-1)}-\boldsymbol{\alpha}(\mathbf{x}) \\
& -\boldsymbol{\beta}(\mathbf{x}) \mathbf{u}-\widehat{\Delta}\left(\mathbf{x} \mid \widehat{\boldsymbol{\theta}}_{\Delta}^{\mathrm{T}}\right) \\
= & -\chi \boldsymbol{\zeta}+\tilde{\boldsymbol{\theta}}_{\Delta}^{\mathrm{T}} \boldsymbol{\vartheta}_{\Delta}(\mathbf{x})+\boldsymbol{\varepsilon}-\widehat{v} \tanh \left(\frac{\zeta}{c}\right),
\end{aligned}
$$

where $\widetilde{\boldsymbol{\theta}}_{\Delta}=\boldsymbol{\theta}_{\Delta}^{*}-\widehat{\boldsymbol{\theta}}_{\Delta}$ is an adjustable parameter vector.
The IFDO-PSMC is proposed as

$$
\begin{aligned}
& \mathbf{u}=- T^{-1} \boldsymbol{\beta}(x)^{-1} \\
& \cdot\left(\mathbf{s}(t)+T\left(\boldsymbol{\alpha}(x)+\widehat{\boldsymbol{\theta}}_{\Delta}^{\mathrm{T}} \boldsymbol{\vartheta}_{\Delta}(\mathbf{x})\right.\right. \\
&\left.\left.\quad-\mathbf{y}_{\mathbf{c}}^{(\boldsymbol{\rho})}+\mathbf{z}+\widehat{v} \tanh \left(\frac{\mathbf{s}(t)}{c}\right)\right)\right) .
\end{aligned}
$$

Invoking (16) and (47), we have

$$
\begin{aligned}
\dot{\mathbf{s}} & =\mathbf{y}^{(\boldsymbol{\rho})}-\mathbf{y}_{\mathbf{c}}^{(\boldsymbol{\rho})}+\mathbf{z}=\boldsymbol{\alpha}(\mathbf{x})+\boldsymbol{\beta}(\mathbf{x}) \mathbf{u}+\Delta(\mathbf{x})-\mathbf{y}_{\mathbf{c}}^{(\boldsymbol{\rho})}+\mathbf{z} \\
& =-T^{-1} \mathbf{s}+\widetilde{\boldsymbol{\theta}}_{\Delta}^{\mathrm{T}} \boldsymbol{\vartheta}_{\Delta}(\mathbf{x})+\boldsymbol{\varepsilon}-\widehat{v} \tanh \left(\frac{\mathbf{s}}{c}\right) .
\end{aligned}
$$

Invoking (46) and (48), the augmented system is obtained as

$$
\dot{\boldsymbol{\zeta}}=\mathbf{A} \boldsymbol{\varsigma}+\mathbf{B}\left(\tilde{\boldsymbol{\theta}}_{\Delta}^{\mathrm{T}} \boldsymbol{\vartheta}_{\Delta}(\mathbf{x})+\boldsymbol{\varepsilon} \widehat{v} \tanh \left(\frac{\boldsymbol{\varsigma}^{\mathrm{T}} \mathbf{B}}{c}\right)\right)
$$

where $\boldsymbol{\varsigma}=\left(\boldsymbol{\zeta}^{\mathrm{T}}, \mathbf{s}^{\mathrm{T}}\right)^{\mathrm{T}}, \mathbf{A}=\operatorname{diag}\left(-\chi \mathbf{I}_{m},-T^{-1} \mathbf{I}_{m}\right)$, and $\mathbf{B}=$ $\left(\mathbf{I}_{m}, \mathbf{I}_{m}\right)^{\mathrm{T}}$.

Theorem 15. Assume that the disturbance of the system (9) is monitored by the system (49), and the system (9) is controlled by (47). If the adjustable parameter vector of IFDO is tuned by (50) and the approximate error compensation parameter is tuned by (51), the augmented error $\boldsymbol{\varsigma}$ is uniformly ultimately bounded within an arbitrarily small region:

$$
\begin{aligned}
\dot{\hat{\boldsymbol{\theta}}}_{\Delta} & =\operatorname{Proj}\left[\lambda \boldsymbol{\vartheta}_{\Delta}(\mathbf{x}) \boldsymbol{\varsigma}^{\mathrm{T}} \mathbf{B}\right] \\
& =\lambda \boldsymbol{\vartheta}_{\Delta}(\mathbf{x}) \boldsymbol{\varsigma}^{\mathrm{T}}-I_{\theta} \lambda \frac{\boldsymbol{\varsigma}^{\mathrm{T}} \mathbf{B} \widehat{\boldsymbol{\theta}}_{\Delta}^{\mathrm{T}} \boldsymbol{\vartheta}_{\Delta}(\mathbf{x})}{\left\|\widehat{\boldsymbol{\theta}}_{\Delta}\right\|^{2}} \widehat{\boldsymbol{\theta}}_{\Delta}, \\
\dot{\hat{v}} & =\eta\left\|\boldsymbol{\varsigma}^{\mathrm{T}} \mathbf{B}\right\|
\end{aligned}
$$

where

$$
\begin{aligned}
& I_{\theta} \\
& = \begin{cases}0, & \text { if }\left\|\widehat{\boldsymbol{\theta}}_{\Delta}\right\|<m_{\theta}, \text { or }\left\|\widehat{\boldsymbol{\theta}}_{\Delta}\right\|=m_{\theta}, \quad \boldsymbol{c}^{\mathrm{T}} \mathbf{B} \widehat{\boldsymbol{\theta}}_{\Delta}^{\mathrm{T}} \boldsymbol{\vartheta}_{\Delta}(\mathbf{x}) \leq 0 \\
1, & \text { if }\left\|\widehat{\boldsymbol{\theta}}_{\Delta}\right\|=m_{\theta}, \quad \boldsymbol{\varsigma}^{\mathrm{T}} \mathbf{B} \widehat{\boldsymbol{\theta}}_{\Delta}^{\mathrm{T}} \boldsymbol{\vartheta}_{\Delta}(\mathbf{x})>0 .\end{cases}
\end{aligned}
$$

Proof. Consider the Lyapunov function candidate:

$$
V=\frac{1}{2} \boldsymbol{\varsigma}^{\mathrm{T}} \boldsymbol{\varsigma}+\frac{1}{2 \lambda} \widetilde{\boldsymbol{\theta}}_{\Delta}^{\mathrm{T}} \widetilde{\boldsymbol{\theta}}_{\Delta}+\frac{1}{2 \eta} \widetilde{v}^{2},
$$


where $\widetilde{v}=\widehat{v}-\bar{\varepsilon}$. Invoking (49), (50), and (51), the time derivative of $V$ is

$$
\begin{aligned}
& \dot{V}=\boldsymbol{\varsigma}^{\mathrm{T}} \dot{\boldsymbol{\xi}}+\frac{1}{\lambda} \widetilde{\boldsymbol{\theta}}_{\Delta}^{\mathrm{T}} \dot{\tilde{\boldsymbol{\theta}}}_{\Delta}+\frac{1}{\eta} \widetilde{v} \dot{\vec{v}}=\boldsymbol{\zeta}^{\mathrm{T}} \dot{\boldsymbol{\zeta}}+\mathbf{s}^{\mathrm{T}} \dot{\boldsymbol{s}}+\frac{1}{\lambda} \widetilde{\boldsymbol{\theta}}_{\Delta}^{\mathrm{T}} \dot{\tilde{\boldsymbol{\theta}}}_{\Delta}+\frac{1}{\eta} \widetilde{v} \dot{v} \\
& =\boldsymbol{\zeta}^{\mathrm{T}}\left(-\chi \boldsymbol{\zeta}+\widetilde{\boldsymbol{\theta}}_{\Delta}^{\mathrm{T}} \boldsymbol{\vartheta}_{\Delta}(\mathbf{x})+\boldsymbol{\varepsilon}-\widehat{v} \tanh \left(\frac{\boldsymbol{\zeta}}{c}\right)\right) \\
& +\mathbf{s}^{\mathrm{T}}\left(-T^{-1} \mathbf{s}+\widetilde{\boldsymbol{\theta}}_{\Delta}^{\mathrm{T}} \boldsymbol{\vartheta}_{\Delta}(\mathbf{x})+\boldsymbol{\varepsilon}\right) \\
& -\widehat{v} \tanh \left(\frac{\mathbf{s}}{c}\right)-\frac{1}{\lambda} \tilde{\boldsymbol{\theta}}_{\Delta}^{\mathrm{T}} \operatorname{Proj}\left[\lambda \boldsymbol{\vartheta}_{\Delta}(\mathbf{x}) \boldsymbol{\varsigma}^{\mathrm{T}} \mathbf{B}\right]+\frac{1}{\eta} \tilde{v} \dot{\tilde{v}} \\
& =\boldsymbol{\zeta}^{\mathrm{T}}\left(-\chi \boldsymbol{\zeta}+\widetilde{\boldsymbol{\theta}}_{\Delta}^{\mathrm{T}} \boldsymbol{\vartheta}_{\Delta}(\mathbf{x})+\boldsymbol{\varepsilon}-\widehat{v} \tanh \left(\frac{\zeta}{c}\right)\right) \\
& +\mathbf{s}^{\mathrm{T}}\left(-T^{-1} \mathbf{s}+\widetilde{\boldsymbol{\theta}}_{\Delta}^{\mathrm{T}} \boldsymbol{\vartheta}_{\Delta}(\mathbf{x})+\boldsymbol{\varepsilon}\right) \\
& -\widehat{v} \tanh \left(\frac{\mathbf{s}}{c}\right)-\widetilde{\boldsymbol{\theta}}_{\Delta}^{\mathrm{T}} \boldsymbol{\vartheta}_{\Delta}(\mathbf{x}) \boldsymbol{\varsigma}^{\mathrm{T}} \\
& +\widetilde{\boldsymbol{\theta}}_{\Delta}^{\mathrm{T}} I_{\boldsymbol{\theta}} \frac{\boldsymbol{c}^{\mathrm{T}} \mathbf{B} \widehat{\boldsymbol{\theta}}_{\Delta}^{\mathrm{T}} \boldsymbol{\vartheta}_{\Delta}(\mathbf{x})}{\left\|\widehat{\boldsymbol{\theta}}_{\Delta}\right\|^{2}} \widehat{\boldsymbol{\theta}}_{\Delta}+\frac{1}{\eta} \tilde{v} \dot{v} \\
& \leq \zeta^{\mathrm{T}}\left(-\chi \zeta+\tilde{\boldsymbol{\theta}}_{\Delta}^{\mathrm{T}} \boldsymbol{\vartheta}_{\Delta}(\mathbf{x})+\boldsymbol{\varepsilon}-\widehat{v} \tanh \left(\frac{\zeta}{c}\right)\right) \\
& +\mathbf{s}^{\mathrm{T}}\left(-T^{-1} \mathbf{s}+\widetilde{\boldsymbol{\theta}}_{\Delta}^{\mathrm{T}} \boldsymbol{\vartheta}_{\Delta}(\mathbf{x})+\boldsymbol{\varepsilon}-\widehat{v} \tanh \left(\frac{\mathbf{s}}{c}\right)\right) \\
& -\widetilde{\boldsymbol{\theta}}_{\Delta}^{\mathrm{T}} \boldsymbol{\vartheta}_{\Delta}(\mathbf{x}) \boldsymbol{\varsigma}^{\mathrm{T}}+\frac{1}{\eta} \tilde{v} \dot{\tilde{v}} \\
& \leq \boldsymbol{\zeta}^{\mathrm{T}}\left(-\chi \boldsymbol{\zeta}+\widetilde{\boldsymbol{\theta}}_{\Delta}^{\mathrm{T}} \boldsymbol{\vartheta}_{\Delta}(\mathbf{x})+\boldsymbol{\varepsilon}-\widehat{v} \tanh \left(\frac{\boldsymbol{\zeta}}{c}\right)\right) \\
& +\mathbf{s}^{\mathrm{T}}\left(-T^{-1} \mathbf{s}+\widetilde{\boldsymbol{\theta}}_{\Delta}^{\mathrm{T}} \boldsymbol{\vartheta}_{\Delta}(\mathbf{x})+\boldsymbol{\varepsilon}-\widehat{v} \tanh \left(\frac{\mathbf{s}}{c}\right)\right) \\
& -\widetilde{\boldsymbol{\theta}}_{\Delta}^{\mathrm{T}} \boldsymbol{\vartheta}_{\Delta}(\mathbf{x}) \boldsymbol{\zeta}-\widetilde{\boldsymbol{\theta}}_{\Delta}^{\mathrm{T}} \boldsymbol{\vartheta}_{\Delta}(\mathbf{x}) \mathbf{s}+\frac{1}{\eta} \tilde{v} \dot{\tilde{v}} \\
& \leq \boldsymbol{\zeta}^{\mathrm{T}}(-\chi \zeta+\boldsymbol{\varepsilon})+\mathbf{s}^{\mathrm{T}}\left(-T^{-1} \mathbf{s}+\boldsymbol{\varepsilon}\right)-\boldsymbol{\zeta}^{\mathrm{T}} \widehat{v} \tanh \left(\frac{\zeta}{c}\right) \\
& -\mathbf{s}^{\mathrm{T}} \widehat{v} \tanh \left(\frac{\mathbf{s}}{c}\right)+\widetilde{v}\|\boldsymbol{\zeta}\|+\widetilde{v}\|\mathbf{s}\| .
\end{aligned}
$$

According to Lemma 14, we have

$$
\begin{aligned}
& -\zeta^{\mathrm{T}} \widehat{v} \tanh \left(\frac{\zeta}{c}\right) \leq-|\widehat{v}|\|\zeta\|+m \kappa c, \\
& -\mathbf{s}^{\mathrm{T}} \widehat{v} \tanh \left(\frac{\mathbf{s}}{c}\right) \leq-|\widehat{v}|\|\mathbf{s}\|+m \kappa c .
\end{aligned}
$$

Consequently, we obtain

$$
\begin{aligned}
\dot{V} \leq & -\sigma\|\boldsymbol{\zeta}\|^{2}+\boldsymbol{\zeta}^{\mathrm{T}} \boldsymbol{\varepsilon}-T^{-1}\|\mathbf{s}\|^{2}+\mathbf{s}^{\mathrm{T}} \boldsymbol{\varepsilon}-|\widehat{v}|\|\boldsymbol{\zeta}\| \\
& +m \kappa c-|\widehat{v}|\|\mathbf{s}\|+m \kappa c+\widetilde{v}\|\boldsymbol{\zeta}\|+\widetilde{v}\|\mathbf{s}\|
\end{aligned}
$$

$$
\begin{aligned}
\leq & -\sigma\|\zeta\|^{2}+\|\zeta\| \bar{\varepsilon}-T^{-1}\|\mathbf{s}\|^{2}+\|\mathbf{s}\| \bar{\varepsilon}-\widehat{v}\|\zeta\| \\
& +\widetilde{v}\|\zeta\|-\widehat{v}\|\zeta\|+\widetilde{v}\|\mathbf{s}\|+2 m \kappa c \\
\leq & -\sigma\|\zeta\|^{2}-T^{-1}\|\mathbf{s}\|^{2}+2 m \kappa c .
\end{aligned}
$$

If $\|\boldsymbol{\zeta}\|>\sqrt{2 m \kappa c / \chi}$ or $\|\mathbf{s}\|>\sqrt{2 m \kappa c T}, \dot{V}<0$. Therefore, the augmented error $\boldsymbol{\varsigma}$ is uniformly ultimately bounded.

Remark 16. The composite disturbances can be approximated effectively by adjusting the parameter law (50) and (51). If the fuzzy system approximates the composite disturbances accurately, the approximate error compensation will have small effect on compensating the approximate error, and vice versa. Based on this, the approximate precision of FDO can be improved through overall consideration.

\section{Design of Attitude Control of Hypersonic Vehicle}

4.1. Control Strategy. The structure of the IFDO-PSMC flight control system is shown in Figure 1. Considering the strong coupled, nonlinear, and uncertain features of the HV, predictive sliding mode control approach which has distinct merits is applied to the design of the nominal flight control system. To further improve the performance of flight control system, an improved fuzzy disturbance observer is proposed to estimate the composite disturbances. The adjustable parameter law of IFDO can be designed based on Lyapunov theorem to approximate the composite disturbances online. According to the estimated composite disturbances, the compensation controller can be designed and integrated with the nominal controller to track the guidance commands precisely. In order to improve the quality of flight control system, three same command filters are designed to smooth the changing of the guidance commands in three channels. Their transfer functions have the same form as

$$
\frac{x_{r}}{x_{c}}=\frac{2}{x+2}
$$

where $x_{r}$ and $x_{c}$ are the reference model states and the external input guidance commands, respectively.

4.2. Attitude Controller Design. The states of the attitude model (2) are pitch angle $\varphi$, yaw angle $\psi$, rolling angle $\gamma$, pitch rate $\omega_{x}$, yaw rate $\omega_{y}$, and roll rate $\omega_{z}$, and the outputs are selected as pitch angle $\varphi$, yaw angle $\psi$, and rolling angle $\gamma$, which can be written as $\boldsymbol{\Theta}=[\varphi, \psi, \gamma]^{\mathrm{T}}$ and $\boldsymbol{\omega}=\left[\omega_{x}, \omega_{y}, \omega_{z}\right]^{\mathrm{T}}$, and the output states are selected as $\mathbf{y}=[\varphi, \psi, \gamma]^{\mathrm{T}}$; then, the attitude model (2) can be rewritten as

$$
\begin{aligned}
& \dot{\boldsymbol{\Theta}}=\mathbf{F}_{\theta} \boldsymbol{\omega} \\
& \dot{\boldsymbol{\omega}}=\mathbf{J}^{-1} \mathbf{F}_{\omega}+\mathbf{J}^{-1} \mathbf{u},
\end{aligned}
$$




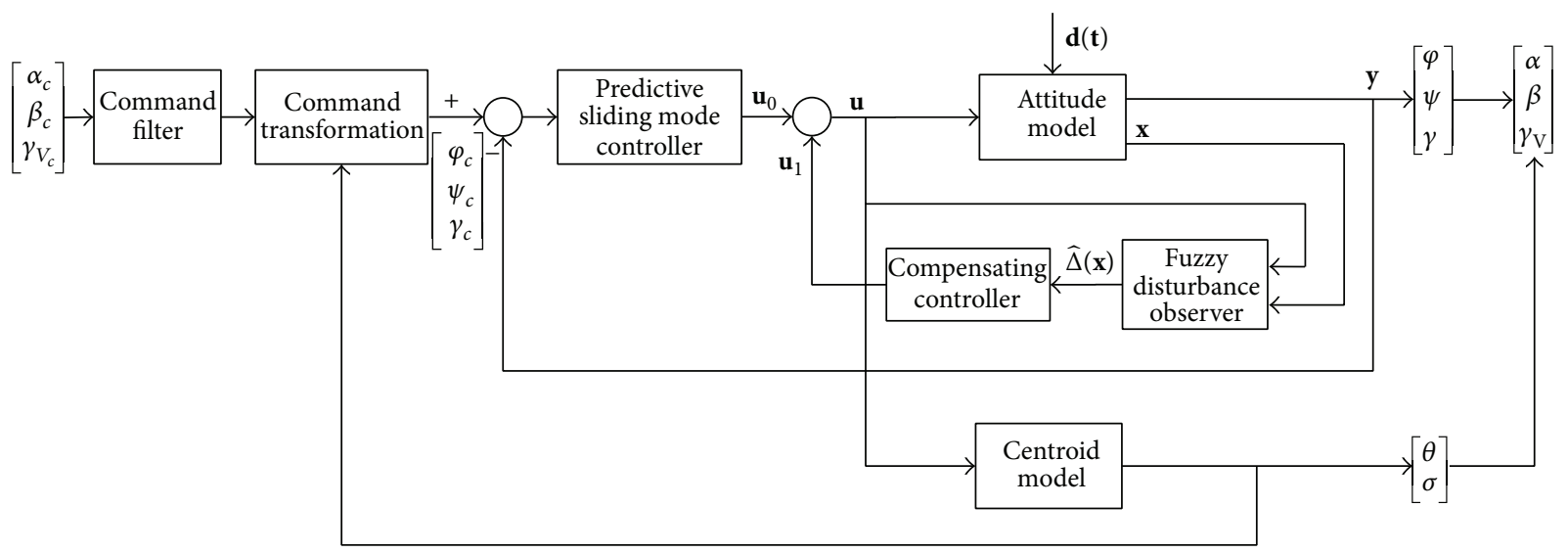

FIGURE 1: IFDO-PSMC based flight control system.

where

$$
\begin{aligned}
\mathbf{F}_{\theta} & =\left[\begin{array}{ccc}
0 & \sin \gamma & \cos \gamma \\
0 & \cos \gamma \sec \varphi & -\sin \gamma \sec \varphi \\
1 & -\tan \varphi \cos \gamma & \tan \varphi \sin \gamma
\end{array}\right], \\
\mathbf{u} & =\left[\begin{array}{l}
M_{x} \\
M_{y} \\
M_{z}
\end{array}\right] \\
\mathbf{F}_{\omega} & =\left[\begin{array}{c}
\left(J_{y}-J_{z}\right) \omega_{z} \omega_{y}-\dot{J}_{x} \omega_{x} \\
\left(J_{z}-J_{x}\right) \omega_{x} \omega_{z}-\dot{J}_{y} \omega_{y} \\
\left(J_{x}-J_{y}\right) \omega_{x} \omega_{y}-\dot{J}_{z} \omega_{z}
\end{array}\right] \\
\mathbf{J} & =\left[\begin{array}{ccc}
J_{x} & 0 & 0 \\
0 & J_{y} & 0 \\
0 & 0 & J_{z}
\end{array}\right] .
\end{aligned}
$$

According to the PSMC approach, (58) can be derived as

$$
\ddot{\mathbf{y}}=\alpha(\mathbf{x})+\beta(\mathbf{x}) \mathbf{u}
$$

where

$$
\begin{aligned}
\boldsymbol{\alpha}(\mathbf{x}) & \\
= & {\left[\begin{array}{ccc}
0 & \sin \gamma & \cos \gamma \\
0 & \cos \gamma \sec \varphi & -\sin \gamma \sec \varphi \\
1 & -\tan \varphi \cos \gamma & \tan \varphi \sin \gamma
\end{array}\right] } \\
& \cdot\left[\begin{array}{ccc}
J_{x}^{-1} & 0 & 0 \\
0 & J_{y}^{-1} & 0 \\
0 & 0 & J_{z}^{-1}
\end{array}\right] \\
& \cdot\left[\begin{array}{c}
\left(J_{y}-J_{z}\right) \omega_{z} \omega_{y}-\dot{J}_{x} \omega_{x} \\
\left(J_{z}-J_{x}\right) \omega_{x} \omega_{z}-\dot{J}_{y} \omega_{y} \\
\left(J_{x}-J_{y}\right) \omega_{x} \omega_{y}-\dot{J}_{z} \omega_{z}
\end{array}\right]
\end{aligned}
$$

$$
\begin{gathered}
+\left[\begin{array}{c}
\dot{\gamma}\left(\omega_{y} \cos \gamma-\omega_{z} \sin \gamma\right) \\
\dot{\varphi}\left(\omega_{y} \cos \gamma-\omega_{z} \sin \gamma\right) \tan \varphi \sec \varphi \\
-\dot{\gamma}\left(\omega_{y} \sin \gamma+\omega_{z} \cos \gamma\right) \sec \varphi \\
\dot{\gamma}\left(\omega_{y} \sin \gamma+\omega_{z} \cos \gamma\right) \tan \varphi \\
-\dot{\varphi}\left(\omega_{y} \cos \gamma-\omega_{z} \sin \gamma\right) \sec ^{2} \varphi
\end{array}\right], \\
\boldsymbol{\beta}(\mathbf{x})=\left[\begin{array}{ccc}
0 & \sin \gamma & \cos \gamma \\
0 & \cos \gamma \sec \varphi & -\sin \gamma \sec \varphi \\
1 & -\tan \varphi \cos \gamma & \tan \varphi \sin \gamma
\end{array}\right]\left[\begin{array}{ccc}
J_{x}^{-1} & 0 & 0 \\
0 & J_{y}^{-1} & 0 \\
0 & 0 & J_{z}^{-1}
\end{array}\right] .
\end{gathered}
$$

Then, the sliding surface can be selected as

$$
\mathbf{s}(t)=\dot{\mathbf{e}}+K \mathbf{e},
$$

where $\mathbf{e}=\mathbf{y}-\mathbf{y}_{c}$ is the attitude tracking error vector, $\mathbf{y}_{c}$ is attitude command vector $\left[\varphi_{c}, \psi_{c}, \gamma_{c}\right]^{\mathrm{T}}$ transformed from the input attitude command vector $\left[\alpha_{c}, \beta_{c}, \gamma_{V_{c}}\right]^{\mathrm{T}}$, and $K$ is the parameter matrix which must make the polynomial (62) Hurwitz stable. Define $\mathbf{z}$ as

$$
\mathbf{z}=K \dot{\mathbf{e}} .
$$

In addition,

$$
\mathbf{Y}_{\mathbf{c} \rho}=\left[\ddot{\varphi}_{c}, \ddot{\psi}_{c}, \ddot{\gamma}_{c}\right]^{\mathrm{T}}
$$

Substituting (61), (62), (63), and (64) into (20), the nominal predictive sliding mode flight controller which omits the composite disturbances $\Delta(\mathbf{x})$ is proposed as

$$
\mathbf{u}_{0}=-T^{-1} \boldsymbol{\beta}(\mathbf{x})^{-1}\left(\mathbf{s}(t)-T\left(\boldsymbol{\alpha}(\mathbf{x})-\mathbf{Y}_{\mathbf{c} \boldsymbol{\rho}}+\mathbf{z}\right)\right) .
$$

In order to improve performance of the flight control system, the adaptive compensation controller based on IFDO is designed as

$$
\mathbf{u}_{1}=-T^{-1} \boldsymbol{\beta}(\mathbf{x})^{-1} \widehat{\Delta}(\mathbf{x}),
$$

where $\widehat{\Delta}(\mathbf{x})$ is the approximate composite disturbances for $\Delta(\mathbf{x})$. 


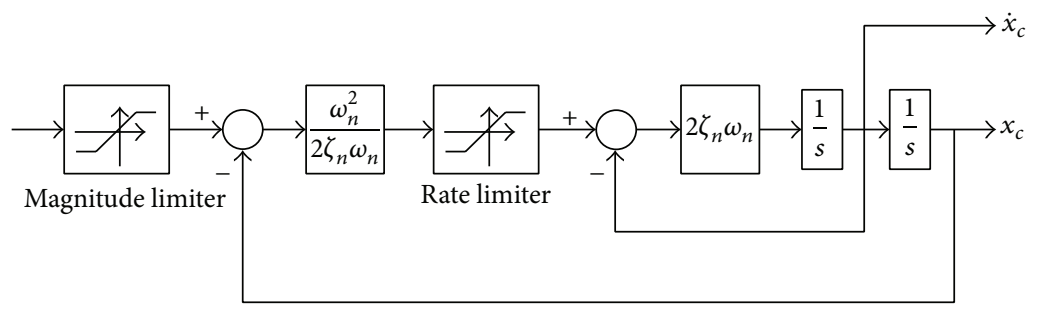

Figure 2: Actuator model.

Accordingly, a fuzzy system can be designed to approximate the composite disturbances $\Delta(\mathbf{x})$. First, each state is divided into five fuzzy sets, namely, $A_{1}^{i}$ (negative big), $A_{2}^{i}$ (negative small), $A_{3}^{i}$ (zero), $A_{4}^{i}$ (positive small), and $A_{5}^{i}$ (positive big), where $i=1,2,3$ represent $\varphi, \psi$, and $\gamma$. Based on the Gaussian membership function, 25 if-then fuzzy rules are used to approximate the composite disturbances $\widehat{\delta}_{1}, \widehat{\delta}_{2}$, and $\widehat{\delta}_{3}$, respectively.

Considering the adaptive parameter law (50) and (51), the approximate composite disturbances can be expressed as

$$
\widehat{\Delta}(\mathbf{x})=\left[\widehat{\delta}_{1}, \widehat{\delta}_{2}, \widehat{\delta}_{3}\right]^{\mathrm{T}}=\widehat{\boldsymbol{\theta}}_{\Delta}^{\mathrm{T}} \boldsymbol{\vartheta}_{\Delta}(\mathbf{x})
$$

where $\widehat{\boldsymbol{\theta}}_{\Delta}^{\mathrm{T}}=\operatorname{diag}\left\{\widehat{\boldsymbol{\theta}}_{1}^{\mathrm{T}}, \widehat{\boldsymbol{\theta}}_{2}^{\mathrm{T}}, \widehat{\boldsymbol{\theta}}_{3}^{\mathrm{T}}\right\}$ is the adjustable parameter vector and $\boldsymbol{\vartheta}_{\Delta}(\mathbf{x})=\left[\boldsymbol{\vartheta}_{1}(\mathbf{x})^{\mathrm{T}}, \boldsymbol{\vartheta}_{2}(\mathbf{x})^{\mathrm{T}}, \boldsymbol{\vartheta}_{3}(\mathbf{x})^{\mathrm{T}}\right]^{\mathrm{T}}$ is a fuzzy basis function vector related to the membership functions; hence, the $\mathrm{HV}$ attitude control based on the IFDO can be written as

$$
\mathbf{u}=\mathbf{u}_{0}+\mathbf{u}_{1}
$$

Theorem 17. Assume that the HV attitude model satisfies Assumptions 3-6 and the sliding surface is selected as (62), which is Hurwitz stable. Moreover, assume that the composite disturbances of the attitude model are monitored by the system (49) and the system is controlled by (68). If the adjustable parameter vector of IFDO is tuned by (50) and the approximate error compensation parameter is tuned by (51), then the attitude tracking error and the disturbance observation error are uniformly ultimately bounded within an arbitrarily small region.

Remark 18. Theorem 17 can be proved similarly as Theorem 15. Herein, it is omitted.

Remark 19. By means of the designed IFDO-PSMC system (68), the proposed HV attitude control system can not only track the guidance commands precisely with strong robustness but also guarantee the stability of the system.

4.3. Actuator Dynamics. The HV attitude system tracks the guidance commands by controlling actuator to generate deflection moments; then, the $\mathrm{HV}$ is driven to change the attitude angles. However, there exist actuator dynamics to generate the input moments $M_{x}, M_{y}$, and $M_{z}$, which are not considered in most of the existing literature. In order to make the study in accordance with the actual case, consider the actuator model expressed as

$$
\frac{x_{c}(s)}{x_{c d}(s)}=\frac{\omega_{n}^{2}}{s^{2}+2 \zeta_{n} \omega_{n}+\omega_{n}^{2}} S_{A}(s) S_{v}(s)
$$

where $\zeta_{n}$ and $\omega_{n}$ are the damping and bandwidth, respectively, $S_{A}(s)$ is the deflection angle limiter, and $S_{v}(s)$ is the deflection rate limiter. Figure 2 shows the actuator model, where $x_{c 0}, x_{c}$, and $\dot{x}_{c}$ are the deflection angle command, virtual deflection angle, and deflection rate, respectively.

\section{Simulation Results Analysis}

To validate the designed HV attitude control system, simulation studies are conducted to track the guidance commands $\left[\alpha_{c}, \beta_{c}, \gamma_{V_{c}}\right]^{\mathrm{T}}$. Assume that the HV flight lies in the cruise phase with the flight altitude $33.5 \mathrm{~km}$ and the flight mach number 15 . The initial attitude and attitude angular velocity conditions are arbitrarily chosen as $\alpha_{0}=\beta_{0}=\gamma_{V_{0}}=0$ and $\omega_{x_{0}}=\omega_{y_{0}}=\omega_{z_{0}}=0$; the initial flight path angle and trajectory angle are $\theta_{0}=\sigma_{0}=0$; the guidance commands are chosen as $\alpha_{c}=5^{\circ}, \beta_{c}=0^{\circ}$, and $\gamma_{V_{c}}=5^{\circ}$, respectively.

The damping $\zeta_{n}$ and bandwidth $\omega_{n}$ of the actuator are 0.707 and $100 \mathrm{rad} / \mathrm{s}$, respectively; the deflection angle limit is $[-20,20]^{\circ}$; the deflection rate limit is $[-50,50]^{\circ} / \mathrm{s}$.

To exhibit the superiority of the proposed scheme, the variation of the aerodynamic coefficients, aerodynamic moment coefficients, atmosphere density, thrust coefficients, and moments of inertia is assumed to be $-50 \%,-50 \%,+50 \%$, $-30 \%$, and $-10 \%$, which are much more rigorous than those considered in [18]. On the other hand, unknown external disturbance moments imposed on the HV are expressed as

$$
\begin{aligned}
& d_{1}(t)=10^{5} \sin (2 t) \mathrm{N} \cdot \mathrm{m} \\
& d_{2}(t)=2 \times 10^{5} \sin (2 t) \mathrm{N} \cdot \mathrm{m} \\
& d_{3}(t)=10^{6} \sin (2 t) \mathrm{N} \cdot \mathrm{m} .
\end{aligned}
$$

The simulation time is set to be $20 \mathrm{~s}$ and the update step of the controller is $0.01 \mathrm{~s}$. The predictive sliding mode controller design parameters are chosen as $T=0.1$ and $K=\operatorname{diag}(2,50,4)$; the IFDO design parameters are chosen as $\lambda=275, \chi=15, \eta=95$, and $c=0.25$. In addition, the membership functions of the fuzzy system are chosen as 


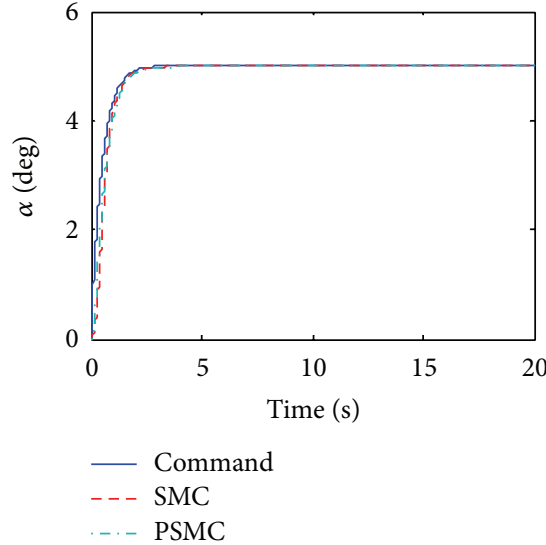

(a)

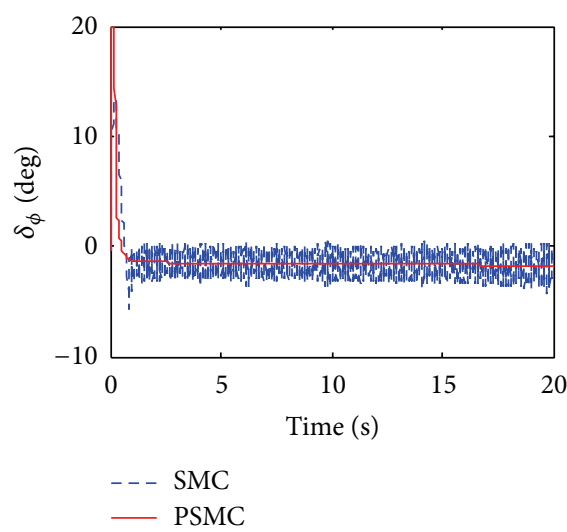

(d)

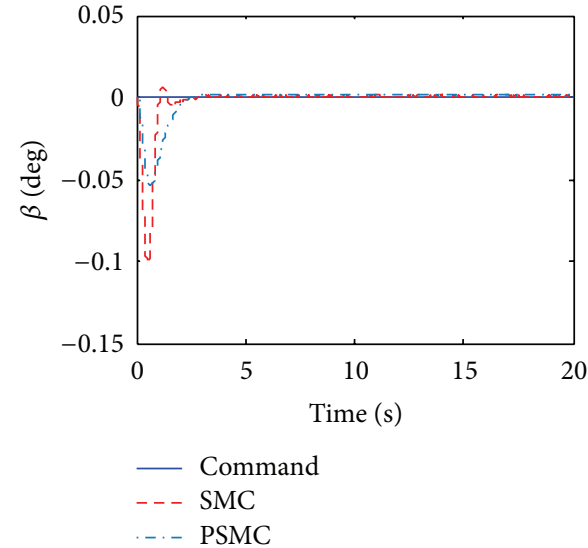

(b)

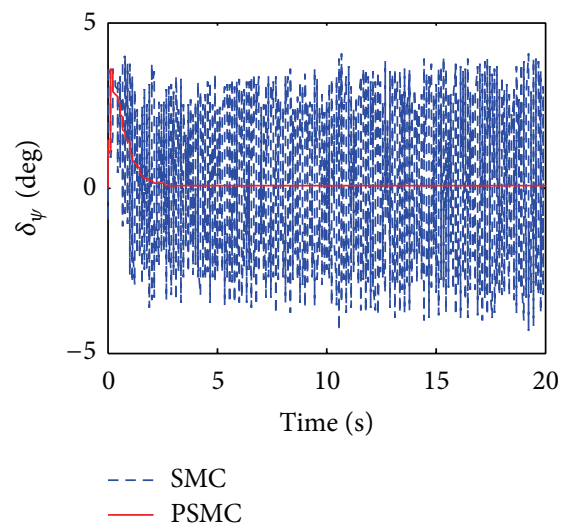

(e)

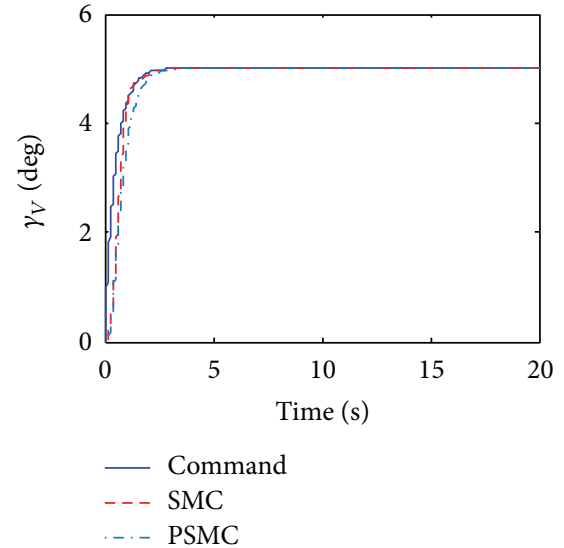

(c)

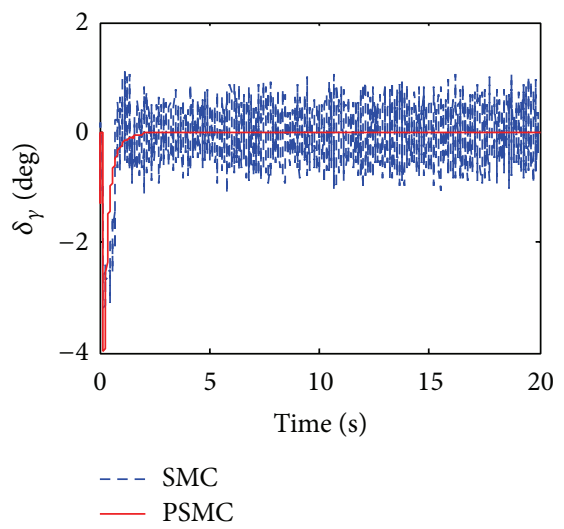

(f)

FIGURE 3: Comparison of tracking curves and control inputs under SMC and PSMC in nominal condition.

Gaussian functions expressed as (71). The simulation results are shown in Figures 3, 4, and 5. Consider

$$
\begin{aligned}
& \mu_{A_{j}^{1}}\left(x_{j}\right)=\exp \left[-\left(\frac{\left(x_{j}+\pi / 6\right)}{(\pi / 24)}\right)^{2}\right], \\
& \mu_{A_{j}^{2}}\left(x_{j}\right)=\exp \left[-\left(\frac{\left(x_{j}+\pi / 12\right)}{(\pi / 24)}\right)^{2}\right], \\
& \mu_{A_{j}^{3}}\left(x_{j}\right)=\exp \left[-\left(\frac{x_{j}}{(\pi / 24)}\right)^{2}\right], \\
& \mu_{A_{j}^{4}}\left(x_{j}\right)=\exp \left[-\left(\frac{\left(x_{j}-\pi / 12\right)}{(\pi / 24)}\right)^{2}\right], \\
& \mu_{A_{j}^{5}}\left(x_{j}\right)=\exp \left[-\left(\frac{\left(x_{j}-\pi / 6\right)}{(\pi / 24)}\right)^{2}\right] .
\end{aligned}
$$

Figure 3 presents the simulation results under PSMC and SMC without considering system uncertainties and external disturbance. Figures 3(a) 3(c) show the results of tracking the guidance commands under PSMC and SMC. As shown, the guidance commands of PSMC and SMC can be tracked both quickly and precisely. Figures 3(d) 3(f) show the control inputs under PSMC and SMC. It can be observed that there exists distinct chattering of SMC while the results of PSMC are smooth owing to the outstanding optimization performance of the predictive control. The simulation results in Figure 3 indicate that the PSMC is more effective than $\mathrm{SMC}$ for system without considering system uncertainties and external disturbances.

Figure 4 shows the simulation results under PSMC and SMC with considering system uncertainties, where the results are denoted as those in Figure 4. From Figures 4(a) 4(c), it can be seen that the flight control system adopted SMC and PSMC can track the commands well in spite of system uncertainties which proves strong robustness of SMC. Meanwhile, it is obvious that SMC takes more time to achieve convergence and the control inputs become larger than that in Figure 4. However, PSMC can still perform as well as that in Figure 3 with the settling time increasing slightly and the 


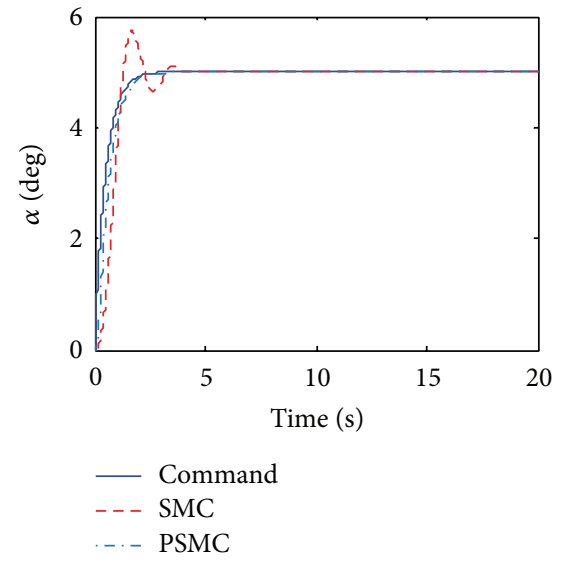

(a)

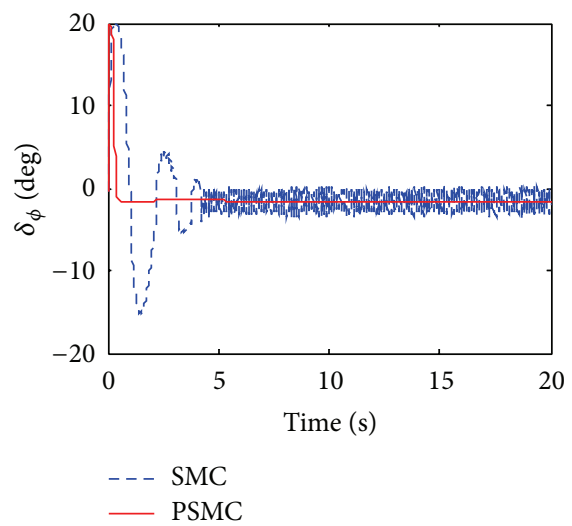

(d)

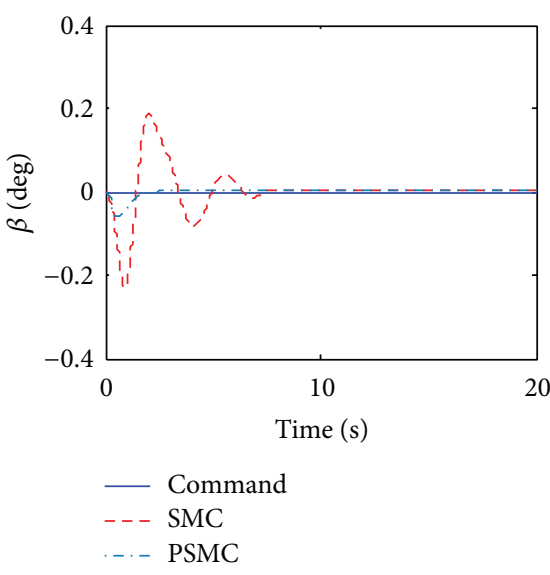

(b)

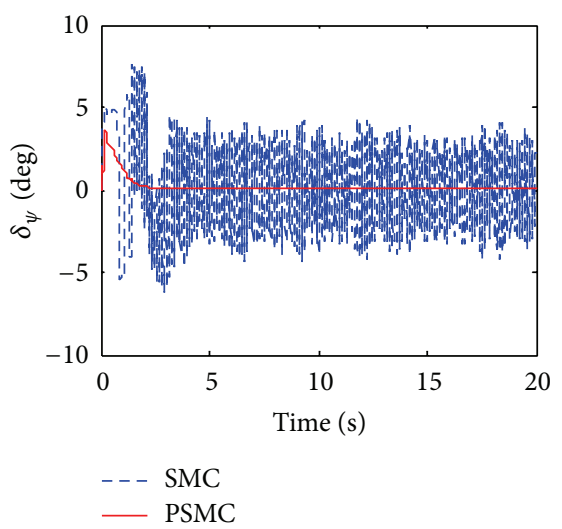

(e)

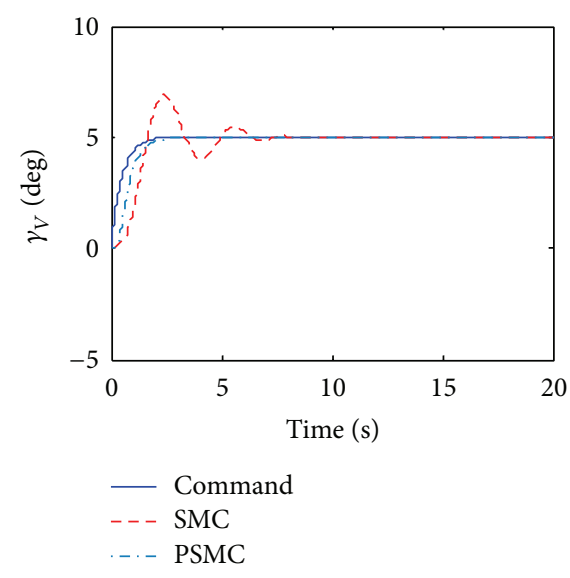

(c)

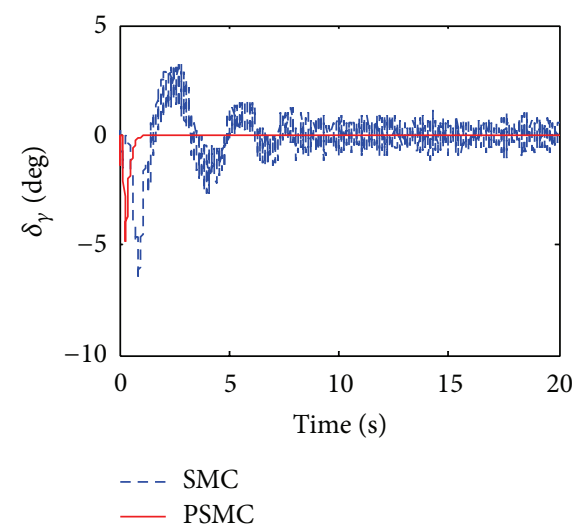

(f)

FIGURE 4: Comparison of tracking curves and control inputs under SMC and PSMC in the presence of system uncertainties.

control inputs almost remain the same. In accordance with Figures $4(\mathrm{~d}) \sim 4(\mathrm{f})$, we can observe that distinct chattering is also caused by SMC while the results of PSMC are smooth. It can be summarized that PSMC is more robust and more accurate than SMC for system with uncertain model.

Figure 5 gives the simulation results under PSMC, FDOPSMC, and IFDO-PSMC in consideration of system uncertainties and external disturbances. Figures 5(a) 5(c) show the results of tracking the guidance commands. It can be observed that PSMC cannot track the guidance commands in the presence of big external disturbances while FDO-PSMC and IFDO-PSMC can both achieve satisfactory performance, which verifies the effectiveness of FDO in suppressing the influence of system uncertainties and external disturbances. Figures 5(d) 5(f) are the partial enlarged detail corresponding to Figures 5(a) 5(c). As shown, the IFDO-PSMC can track the guidance commands more precisely, which declares stronger disturbance approximate ability of IFDO when compared with FDO. To sum up, the results of Figure 5 indicate that IFDO is effective in dealing with system uncertainties and external disturbances. In addition, the proposed
IFDO-PSMC is applicative for the $\mathrm{HV}$ which has rigorous system uncertainties and strong external disturbances.

It can be concluded from the above-mentioned simulation analysis that the proposed IFDO-PSMC flight control scheme is valid.

\section{Conclusions}

An effective control scheme based on PSMC and IFDO is proposed for the HV with high coupling, serious nonlinearity, strong uncertainty, unknown disturbance, and actuator dynamics. PSMC takes merits of the strong robustness of sliding model control and the outstanding optimization performance of predictive control, which seems to be a very promising candidate for $\mathrm{HV}$ control system design. PSMC and FDO are combined to solve the system uncertainties and external disturbance problems. Furthermore, we improve the FDO by incorporating a hyperbolic tangent function with FDO. Simulation results show that IFDO can better approximate the disturbance than FDO and can assure the 


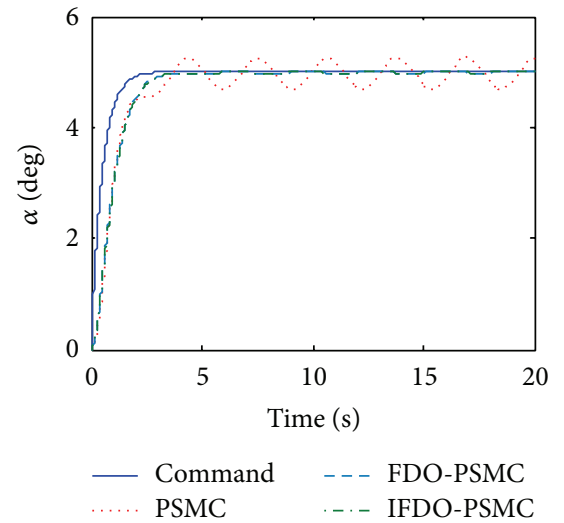

(a)

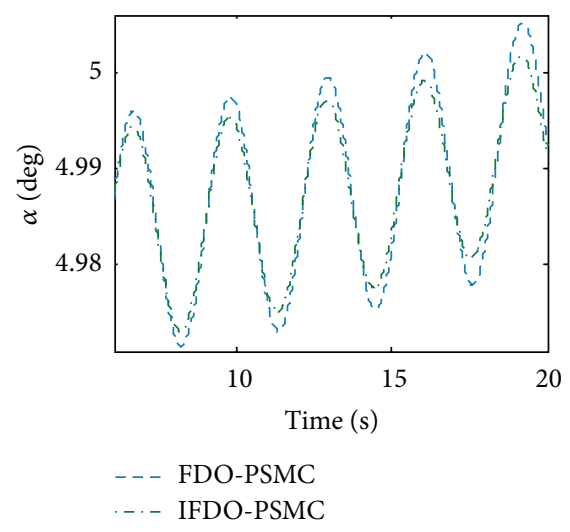

(d)

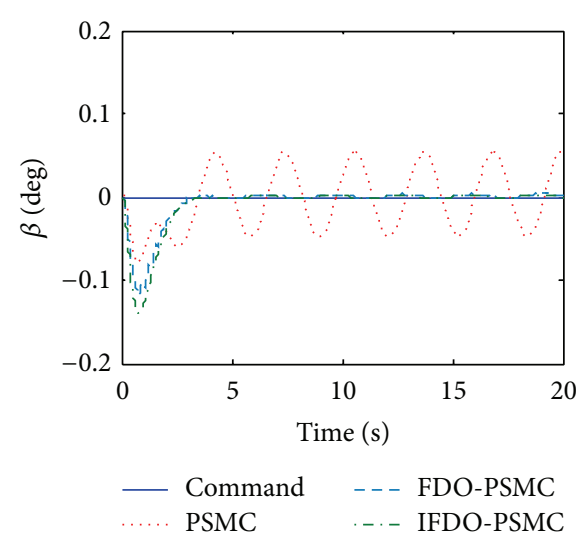

(b)

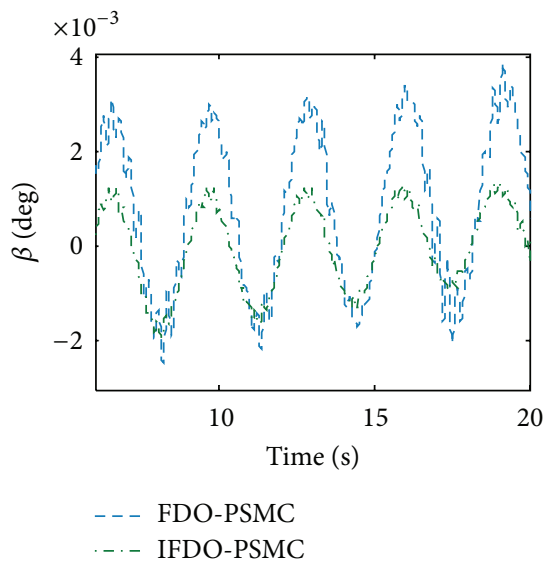

(e)

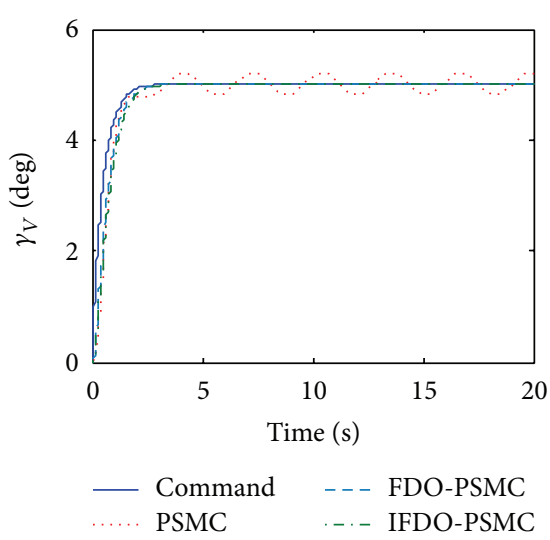

(c)

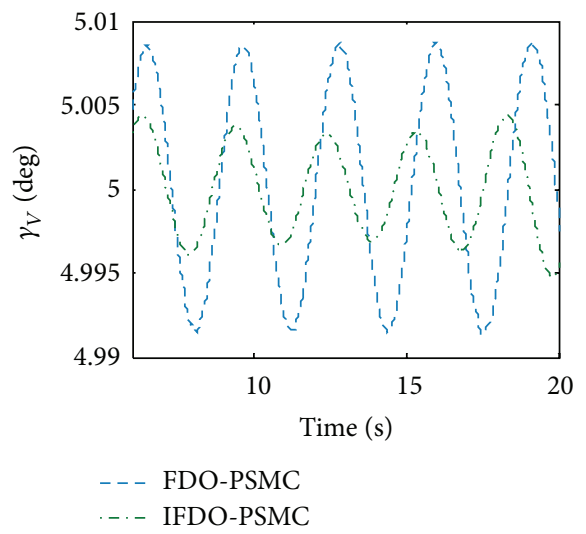

(f)

FIGURE 5: Comparison of tracking curves under PSMC, FDO-PSMC, and IFDO-PSMC in the presence of system uncertainties and external disturbances.

control performance of $\mathrm{HV}$ attitude control system in the presence of rigorous system uncertainties and strong external disturbances.

\section{Conflict of Interests}

The authors declare that there is no conflict of interests regarding the publication of this paper.

\section{References}

[1] E. Tomme and S. Dahl, "Balloons in today's military? An introduction to the near-space concept," Air and Space Power Journal, vol. 19, no. 4, pp. 39-49, 2005.

[2] M. J. Marcel and J. Baker, "Integration of ultra-capacitors into the energy management system of a near space vehicle," in Proceedings of the 5th International Energy Conversion Engineering Conference, June 2007.

[3] M. A. Bolender and D. B. Doman, "Nonlinear longitudinal dynamical model of an air-breathing hypersonic vehicle," Journal of Spacecraft and Rockets, vol. 44, no. 2, pp. 374-387, 2007.

[4] Y. Shtessel, C. Tournes, and D. Krupp, "Reusable launch vehicle control in sliding modes," in Proceedings of the AIAA Guidance, Navigation, and Control Conference, pp. 335-345, 1997.
[5] D. Zhou, C. Mu, and W. Xu, "Adaptive sliding-mode guidance of a homing missile," Journal of Guidance, Control, and Dynamics, vol. 22, no. 4, pp. 589-594, 1999.

[6] F.-K. Yeh, H.-H. Chien, and L.-C. Fu, "Design of optimal midcourse guidance sliding-mode control for missiles with TVC," IEEE Transactions on Aerospace and Electronic Systems, vol. 39, no. 3, pp. 824-837, 2003.

[7] H. Xu, M. D. Mirmirani, and P. A. Ioannou, "Adaptive sliding mode control design for a hypersonic flight vehicle," Journal of Guidance, Control, and Dynamics, vol. 27, no. 5, pp. 829-838, 2004.

[8] Y. N. Yang, J. Wu, and W. Zheng, "Attitude control for a station keeping airship using feedback linearization and fuzzy sliding mode control," International Journal of Innovative Computing, Information and Control, vol. 8, no. 12, pp. 8299-8310, 2012.

[9] Y. N. Yang, J. Wu, and W. Zheng, "Trajectory tracking for an autonomous airship using fuzzy adaptive sliding mode control," Journal of Zhejiang University: Science C, vol. 13, no. 7, pp. 534543, 2012.

[10] Y. Yang, J. Wu, and W. Zheng, "Concept design, modeling and station-keeping attitude control of an earth observation platform," Chinese Journal of Mechanical Engineering, vol. 25, no. 6, pp. 1245-1254, 2012.

[11] Y. Yang, J. Wu, and W. Zheng, "Adaptive fuzzy sliding mode control for robotic airship with model uncertainty and external 
disturbance," Journal of Systems Engineering and Electronics, vol. 23, no. 2, pp. 250-255, 2012.

[12] K.-B. Park and T. Tsuji, "Terminal sliding mode control of second-order nonlinear uncertain systems," International Journal of Robust and Nonlinear Control, vol. 9, no. 11, pp. 769-780, 1999.

[13] S. N. Singh, M. Steinberg, and R. D. DiGirolamo, "Nonlinear predictive control of feedback linearizable systems and flight control system design," Journal of Guidance, Control, and Dynamics, vol. 18, no. 5, pp. 1023-1028, 1995.

[14] L. Fiorentini, A. Serrani, M. A. Bolender, and D. B. Doman, "Nonlinear robust adaptive control of flexible air-breathing hypersonic vehicles," Journal of Guidance, Control, and Dynamics, vol. 32, no. 2, pp. 401-416, 2009.

[15] B. Xu, D. Gao, and S. Wang, "Adaptive neural control based on HGO for hypersonic flight vehicles," Science China Information Sciences, vol. 54, no. 3, pp. 511-520, 2011.

[16] D. O. Sigthorsson, P. Jankovsky, A. Serrani, S. Yurkovich, M. A. Bolender, and D. B. Doman, "Robust linear output feedback control of an airbreathing hypersonic vehicle," Journal of Guidance, Control, and Dynamics, vol. 31, no. 4, pp. 10521066, 2008.

[17] B. Xu, F. Sun, C. Yang, D. Gao, and J. Ren, "Adaptive discretetime controller design with neural network for hypersonic flight vehicle via back-stepping," International Journal of Control, vol. 84, no. 9, pp. 1543-1552, 2011.

[18] P. Wang, G. Tang, L. Liu, and J. Wu, "Nonlinear hierarchystructured predictive control design for a generic hypersonic vehicle," Science China Technological Sciences, vol. 56, no. 8, pp. 2025-2036, 2013.

[19] E. Kim, "A fuzzy disturbance observer and its application to control," IEEE Transactions on Fuzzy Systems, vol. 10, no. 1, pp. 77-84, 2002.

[20] E. Kim, "A discrete-time fuzzy disturbance observer and its application to control," IEEE Transactions on Fuzzy Systems, vol. 11, no. 3, pp. 399-410, 2003.

[21] E. Kim and S. Lee, "Output feedback tracking control of MIMO systems using a fuzzy disturbance observer and its application to the speed control of a PM synchronous motor," IEEE Transactions on Fuzzy Systems, vol. 13, no. 6, pp. 725-741, 2005.

[22] Z. Lei, M. Wang, and J. Yang, "Nonlinear robust control of a hypersonic flight vehicle using fuzzy disturbance observer," Mathematical Problems in Engineering, vol. 2013, Article ID 369092, 10 pages, 2013.

[23] Y. Wu, Y. Liu, and D. Tian, "A compound fuzzy disturbance observer based on sliding modes and its application on flight simulator," Mathematical Problems in Engineering, vol. 2013, Article ID 913538, 8 pages, 2013.

[24] C.-S. Tseng and C.-K. Hwang, "Fuzzy observer-based fuzzy control design for nonlinear systems with persistent bounded disturbances," Fuzzy Sets and Systems, vol. 158, no. 2, pp. 164179, 2007.

[25] C.-C. Chen, C.-H. Hsu, Y.-J. Chen, and Y.-F. Lin, "Disturbance attenuation of nonlinear control systems using an observerbased fuzzy feedback linearization control," Chaos, Solitons \& Fractals, vol. 33, no. 3, pp. 885-900, 2007.

[26] S. Keshmiri, M. Mirmirani, and R. Colgren, "Six-DOF modeling and simulation of a generic hypersonic vehicle for conceptual design studies," in Proceedings of AIAA Modeling and Simulation Technologies Conference and Exhibit, pp. 2004-4805, 2004.
[27] A. Isidori, Nonlinear Control Systems: An Introduction, III, Springer, New York, NY, USA, 1995.

[28] L. X. Wang, "Fuzzy systems are universal approximators," in Proceedings of the IEEE International Conference on Fuzzy Systems, pp. 1163-1170, San Diego, Calif, USA, March 1992.

[29] S. Huang, K. K. Tan, and T. H. Lee, "An improvement on stable adaptive control for a class of nonlinear systems," IEEE Transactions on Automatic Control, vol. 49, no. 8, pp. 1398-1403, 2004.

[30] C. Y. Zhang, Research on Modeling and Adaptive Trajectory Linearization Control of an Aerospace Vehicle, Nanjing University of Aeronautics and Astronautics, 2007. 


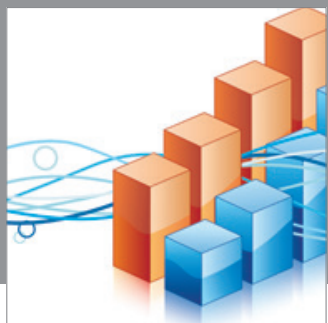

Advances in

Operations Research

mansans

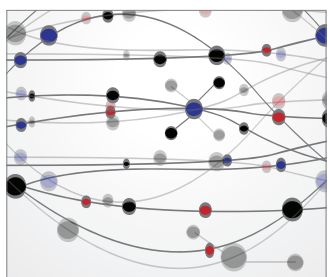

The Scientific World Journal
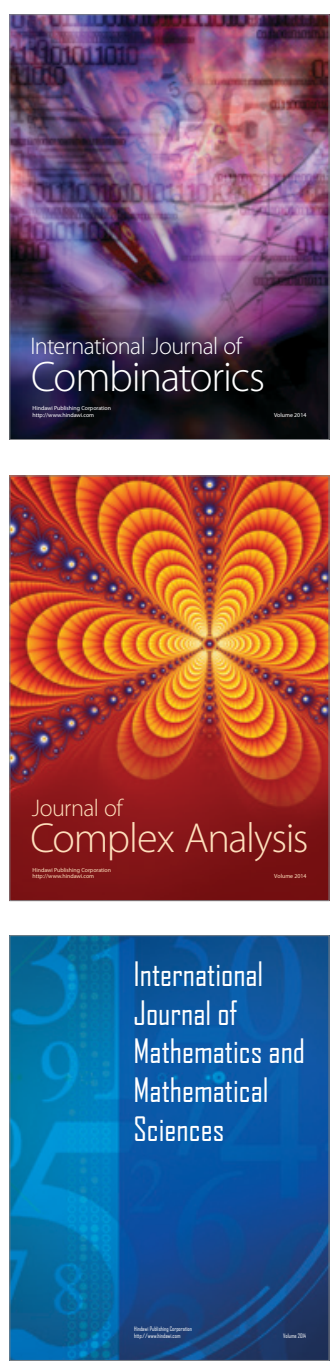
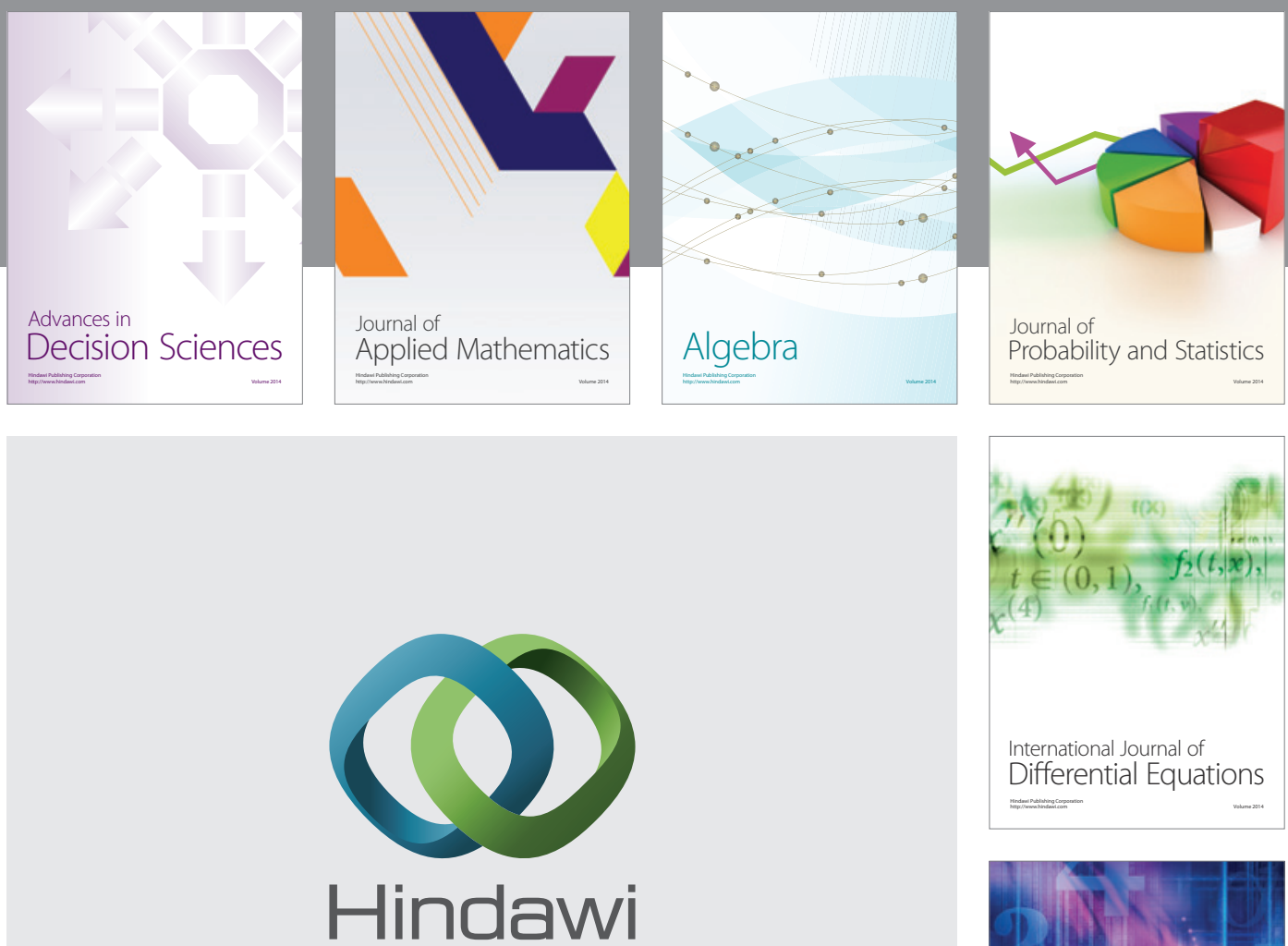

Submit your manuscripts at http://www.hindawi.com
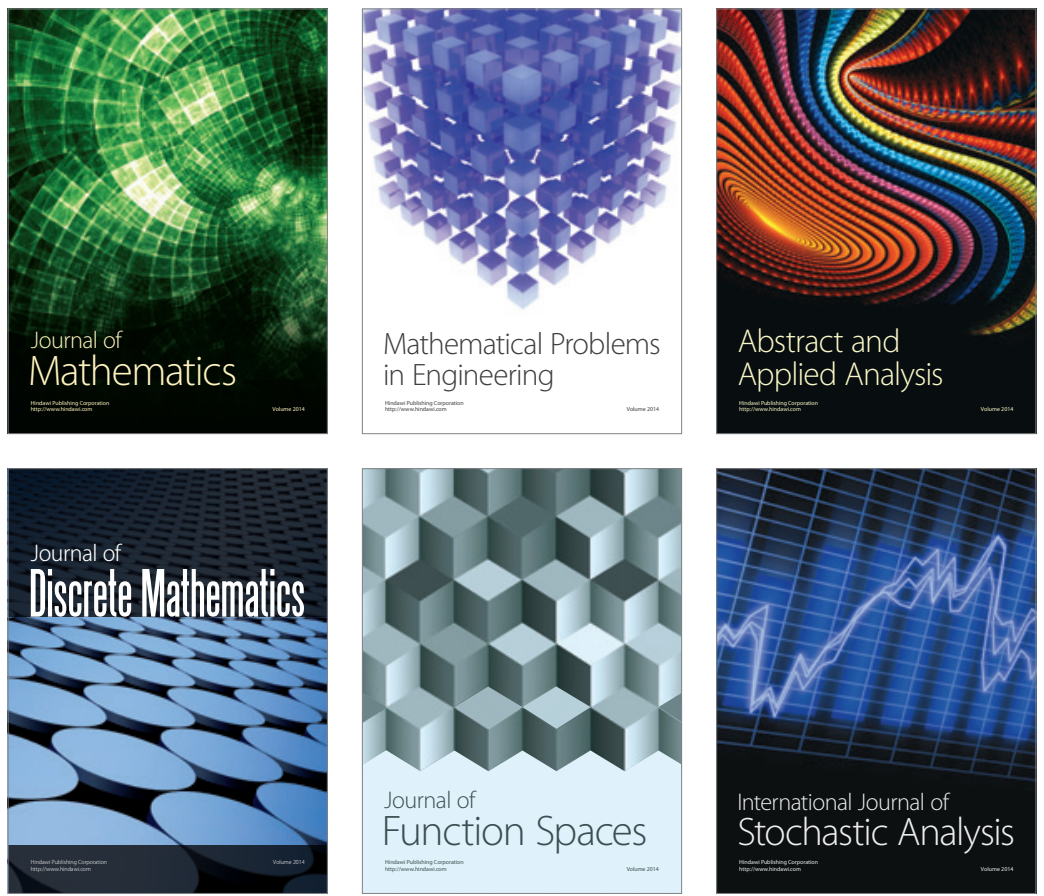

Journal of

Function Spaces

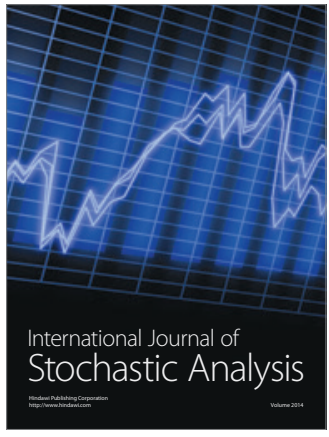

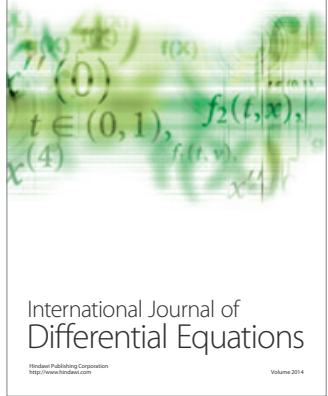
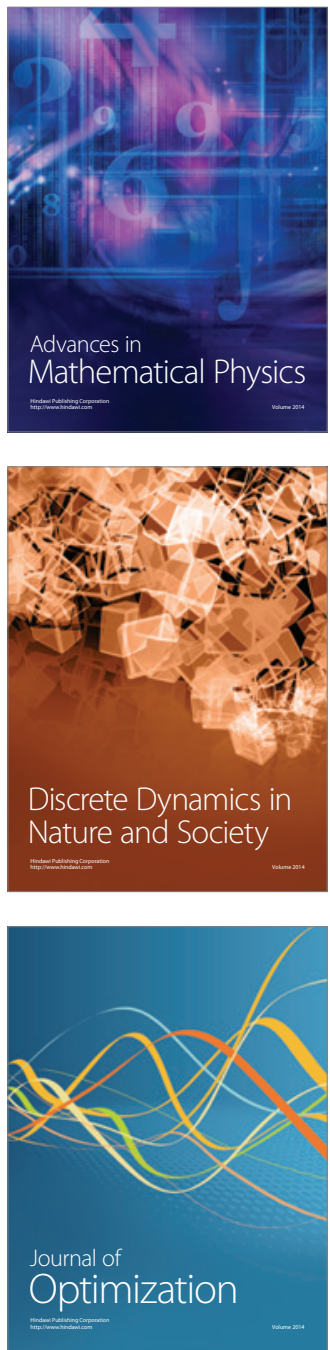